\title{
10. SOLAR ACTIVITY (ACTIVITÉ SOLAIRE)
}

President: Z. Švestka.

Vice-President: J. T. Jefferies.

Organizing Committee: K. O. Kiepenheuer, R. Michard, S. Nagasawa, G. Newkirk, J. Rösch, A. B. Severnyj, M. Waldmeier.

SECRETARY: A. D. Fokker.

The reader is kindly asked to consider this report for some sort of greatly unbalanced equilibrium between the pressure exercised by the steadily increasing flow of papers discussing the vastly extending subject of solar activity, and the equally steady pressure exercised by the editor who insists on having this report twice as short as three years ago. Therefore, some selection of the subjects and papers had to be made, a fairly severe one, since only about one third of all published papers could have been mentioned in the references, and I am well aware that almost everyone who will read the report, could suggest some improvement of the selection scheme. Nevertheless, I believe that the report as it stands, can fulfill its basic purpose, i.e. to give to the readers a general picture on the progress, which has been achieved in the field of solar activity research since the Prague meeting and on some of the research activities which are in progress at the present time.

It is a great pleasure to acknowledge the enormous help I have received from the authors of the individual sections of the Draft Report, as well as from many other members of the Commission, who have very kindly provided me, or the co-authors, with useful information on the recent progress of their work. It is necessary to add that some highly valuable comments made by V. Bumba, J. T. Jefferies, C. Sawyer, E. v. P. Smith, B. Valníček and J. M. Wilcox, have also been included in other chapters than those prepared by themselves.

\section{INTRODUCTION}

One of the most remarkable characteristics of the recent years is an increasing effort to improve the resolving power both of ground-base and space research equipment. The excellent white-light and $\mathrm{H} \alpha$ photographs obtained at Culgoora, Athens, Sacramento Peak and many other stations, several ingenious improvements of solar magnetography, the new Australian and Japanese compound radio interferometers, and X-ray photographs of the Sun, going down to the order of 1 " in resolving power, are outstanding manifestations of this general effort. New solar stations built at water-surrounded sites and the ATM project indicate that this tendency will continue in the coming years. Thus the fine structure of solar active phenomena, detected by the theoreticians in the past years both from spectroscopic and energetic studies, is being attacked now by the solar observers.

Another great progress has been achieved in extending our knowledge of the solar spectrum towards both its ends. Very low frequency radio telescopes on satellites, and X-ray and EUV spectrometers and spectroheliographs, on the OSO satellites in particular, have contributed to this progress. In some research fields, and what I have in mind, are the magnetographic and X-ray studies, in particular, routine studies are being replaced by ingenious improvements in the quality of the obtained results. The continuously increasing sensitivity of receptors has enabled us to detect very small particle events in space and permanent particle emission from specific active regions. Well organized international cooperation has brought some new aspects into the study of complex solar phenomena which, in their consequences, reach far behind the orbit of the Earth.

For other summarizing considerations and all the details the reader is referred to the individual chapters of the Report. Before concluding this very brief introduction, however, I would like to mention two periodicals, which have extensively developed in the past three years and which, 
according to my opinion, have been of great assistance to research workers in the field of solar activity: Solar Physics, the journal starting in January, 1967 (edited by C. de Jager and Z. Švestka, published by D. Reidel Publ. Comp., Dordrecht-Holland), and Solar Geophysical Data, from 1969 divided into two parts, comprising prompt and comprehensive reports respectively (edited by J. Virginia Lincoln, published by ESSA at Boulder, U.S.A.). This second publication, jointly with the Quarterly Bulletin on Solar Activity, provides solar physicists, geophysicists, and space scientists with a very comprehensive selection of data from the field of solar-terrestrial physics, and the work of Miss Lincoln and her co-workers is to be greatly appreciated.

\section{EQUIPMENT AND METHODS}

(The reader is also referred to the "Progress Reports from Solar Institutes", published in the past three years in the journal Solar Physics).

\subsection{Optical observations}

(by B. Valníček, Ondřejov)

The effort to find best seeing conditions for solar work has resulted in the establishment of new solar stations at water-surrounded sites. The great advantage of such establishments has been well demonstrated by Becker (1969) for four different sites in California, and the place for the Aerospace Corporation Observatory was selected on a narrow neck of land that extended into the San Fernando reservoir. Results in $\mathrm{H} \alpha$ and in integrated light are convincing. Similar effect has been reached by the Mt Wilson and Palomar Observatory as a result of a 3-year site survey carried out by Leighton, Howard and Zirin. The new solar observatory is constructed in the middle of Big Bear Lake in the San Bernardino Mountains. In Canada, a peninsula extending into a wide bay of the Ottawa River is under development as a telescope site (Gaizauskas, 1969).

New solar towers are prepared for observation at Kandilli Observatory, with a $60 / 45 \mathrm{~cm}$ coelostat, and at Meudon Observatory, where the work starts in 1970. At Sacramento Peak a new solar tower started operation in autumn 1969 , equipped with many innovations well known in space technology, but not previously incorporated into ground-based solar instruments. The domeless refractor at Capri evoked a similar instrument for the Aerospace Corporation Observatory at San Fernando, where a 24-inch Cassegrain-Gregorian telescope with spectroheliograph has been installed (Becker, 1969). At the Kiev-Goloseevo Observatory a horizontal telescope with 44-cm mirror and $17-\mathrm{m}$ focal length is now in function, with 7-m spectrograph. In Utrecht, a total revision of the horizontal solar spectrograph has been completed by Namba. At Stockholm Observatory a new echelle spectrograph for flare studies is under construction, and at the McMath-Hulbert Observatory the vacuum spectrograph has been digitalized and prepared for realization of a high-dispersion digital atlas of solar spectrum. At Ondřejov the great horizontal telescope is now finished, with $35-\mathrm{m}$ focal length and 10-m spectrograph.

Very characteristic for this epoch of solar research are multiple instruments on solar spars, which make complex work in solar activity studies easily possible. Equipment of this kind is now at the new Solar Station of the Dominion Observatory (equipped to monitor the disk and limb with different filters, and a selected active region in $\mathrm{H} \alpha$ and in integrated light), at the new Big Bear Solar Observatory (with two 10-inch refractors, a 15-inch off-axis Cassegrain system and 9-inch reflecting coronagraph) and at Hawaii (with a $25-\mathrm{cm}$ coronagraph, two $10-\mathrm{cm}$ coronagraphs for work in two different lines and a $15-\mathrm{cm}$ chromospheric telescope). Also the Catania Observatory will be equipped from 1970 with a spar of the same type as at Monte Mario Observatory in Rome. The six-side equatorial spar supports a white light refractor, $\mathrm{H} \alpha$ disc patrol, $\mathrm{H} \alpha$ great size for special events, $\mathrm{H} \alpha$ prominence coronagraph and $\mathrm{K}$ monochromator.

In general, refractors are in renascence for solar work today. At Culgoora a $30-\mathrm{cm}$ refractor equipped with $1 / 8 \AA \mathrm{H} \alpha$ filter is applied to fine chromospheric studies in different parts of the $\mathrm{H} \alpha$ profile. At the National Observatory in Athens a $40-\mathrm{cm}$ refractor with $505-\mathrm{cm}$ focus is working with 
practical resolution of $0 \cdot 6^{\prime \prime}$ in the $\mathrm{H} \alpha$ line. At Ondrejov, a Clark objective, 20-cm aperture and 285 focus, is applied for fine structure studies of the photosphere, in connection with a $0.8 \AA$ Šlc filter. Refractors equipped with $0.25 \AA$ filters are also working at Hawaii and San Fernando.

Several new patrol instruments have been built. For the $\mathrm{H} \alpha$ line at Tel Aviv, Big Bear Lake, Uttar-Pradesh and Kandilli Observatories, and for the CaII K-line in Athens, Uccle and at the Kandilli Observatory again.

Coronagraphs are still in development and many special instruments are now in use for the coronal work. A coronal activity monitor, a branch of coronal polarimeter similar to the coronameter, has been established at Mauna Loa in Hawaii. A balloon-borne coronagraph, for observations of the F-corona in the infrared region near $2 \cdot 2 \mu$ has been described by McQueen (1968). Also at the High Altitude Observatory, Boulder, a coronagraph for the Apollo telescope mount has been constructed, with $3 \cdot 2-\mathrm{cm}$ aperture and $44-\mathrm{cm}$ focus. The corona can be photographed from $1.5 R$ to $6 R$ with the aid of three occulting disks in front of the objective (Newkirk, 1967a). New coronagraphs have been built at the Kislovodsk station of Pulkovo Observatory, with $53-\mathrm{cm}$ objective (Gnevyshev et al., 1967), and at the Norikura Corona Station in Japan, equipped with Littrov spectrograph, focus $7 \mathrm{~m}$, and a $2.5-\mathrm{m}$ echelle-spectrograph. An interesting theoretical study of the coronagraph system has been made by Demarq and Rösch (1967).

A polarization-interference filter with thermo-optically compensated parts, practically eliminating the need for a precise thermostat, has been put into use for coronal observation and investigated by Joffe and Smirnova (1967). A very sophisticated conception of a birefringent filter applying optical rotation of the birefringent plates is proposed by Katzenstein (1968), but its practical use has not been documented.

\subsection{Magnetic field measurements}

(by V. Bumba, Ondřejov)

Because of the urgent need for increased spatial and time resolution in solar observations, a considerable effort in the past three years has gone into the modernization of existing photoelectric magnetographs, improvement of measuring and evaluation methods, and invention of new types of observational devices. At Mt Wilson a more accurate guider, an electro-optic crystal yielding an improved signal-to-noise ratio, an improved Doppler compensator, and a completely automatic data-recording system were introduced (Howard et al., 1968). A new electronic scheme of a magnetograph for simultaneous recording of the longitudinal and vector components of the transversal magnetic field at Crimea was described by Nikulin (1967). Regular observations have been started at Pulkovo with the aid of a six-channel two-slit magnetograph with one photomultiplier and rotating $\lambda / 2$ plate measuring longitudinal as well as transversal fields (Ikhsanov and Platonov, 1967). Deubner and Liedler (1969) have described the new vectormagnetograph of the Fraunhofer Institute at Capri, which measures all components of the magnetic field vector strictly simultaneously. A photographic method is applied which presents longitudinal and transversal field components, vector, azimuth, Doppler shift and intensity in the form of maps. Late in 1967 the Kitt Peak magnetograph expanded to allow transverse measurements, and since the fall of 1969 a 40-channel magnetograph with both longitudinal and transverse output is in operation at the same observatory. It uses advances in fiber optics and micro-circuit technology, with spatial resolution $0.5^{\prime \prime}-2^{\prime \prime}$ for making photoelectric recordings comparable in resolution with spectroheliograms. A new technique for displaying magnetograph observations has been developed by Frazier and Sherrer (1969) and applied to the 12-channel magnetograph at Kitt Peak. Using the data from a raster-scan, a digital "spectroheliogram" is constructed on the face of a cathode ray tube and photographed. The new Gregory-coudé telescope in Locarno (Göttingen station) obtained several improvements with special regard to the magnetic field measurements (Schröter and Wiehr, 1968). At the modified vector magnetograph all four Stokes parameters are measured simultaneously, using a $\lambda / 13.7$ plate and four photomultipliers (Wiehr, 1969). A detailed description of the lambdameter was published by Rayrole (1967) demonstrating some of the advantages of this photographic method. 
At the Rome Observatory a new kind of a very narrow pass-band filter using the atomic-beam techniques in absorption is used as a "Babcock window" for fitting of a magnetograph (Cimino et al., 1968). Giovanelli has announced first preliminary tests of a new type of magnetograph, which uses a high-resolution filter $(\Delta \lambda=0.05 \AA)$ and incorporates three automatically controlled FabryPérot interferometers which can be adjusted to transmit any desired wavelength. The monochromatic solar image is thrown through a $\lambda / 4$-wave plate and a polarizing system and photographed in oppositely circularly polarized components with a short $(\sim 0.3 \mathrm{~s})$ exposure time. Beckers $(1968 a)$ and Ramsey et al. (1968) have described two similar modifications to a birefringent filter, which allow high spatial resolution observations of magnetic and velocity fields in rapid sequences of exposures. A new magnetograph, which is now under construction for the Naval Research Laboratory, Marshall Space Flight Center, and Göttingen Observatory, will be able to produce magnetograms nearly in real time, showing the complete magnetic vector with a resolution of 1" (Brückner, 1968). It will use a birefringent filter and a new SEC vidicon tube which converts the polarization information into electrical currents simultaneously for all picture elements. Digitized electrical signals will be processed by a computer which will prepare the magnetic maps. A real-time video magnetograph using digital enhancement is being constructed by Janssens at the Aerospace Observatory (Becker, 1969).

A solar magnetograph without electronics or any moving parts has been designed by Stenflo $(1968 b)$. Its principle is the use of a subtracting double-dispersion optical system. Stenflo (1969c) also described another method which modulates the light entering the spectroheliograph in the wing of a magnetic-sensitive line by putting alternately $a+$ and $-\lambda / 4$ plate in front of the slit and placing a fixed polarizer after the modulation $\lambda / 4$ plate. In the resulting spectroheliogram the magnetic regions show a fringe pattern where the contrast between the fringes is a direct measure of the field strength. A similar result can be obtained by replacing the varying $\lambda / 4$ plate with a stationary Babinet compensator whose fringes are parallel to the spectrograph dispersion (Beckers and Stenflo, 1969).

Severnyj (1967) was the first who pointed out the problem of large discrepancies between the theoretical calibration curves of a vector magnetograph calculated for an optically thin spectral line in a homogeneous field and those obtained photographically or from visual measurements of Zeeman splitting or from the field strength of a laboratory magnet. The same question was discussed theoretically by Rachkovskij (1967b) who demonstrated that with an assumption of (a) only true line absorption, (b) only line scattering, (c) thin layer line formation, it is not possible to get a good agreement between the theoretical calibration curves and the measured transversal signal. The fact that the use of any theory of spectral line formation in a homogeneous magnetic field cannot explain the discrepancies, has also been demonstrated by Obridko (1968a). In a critical paper, Beckers (1968b) has pointed at an erroneous signal for the Doppler shift which may also lead to incorrect values of the magnetic field strength by several existing magnetographs. Wiehr $(1968,1969)$ has discussed the miscentering by the Doppler compensator, and uncertainties that are associated with the calibration of the response of a vector magnetograph in terms of $\mathrm{H}$ and $\gamma$ using Unno's theory, especially for the line $\lambda 5250$ which is rather sensitive to temperature changes. According to Harvey and Livingston (1969) magnetograph measurements with temperature sensitive lines (such as $F e I \lambda 5250 \AA$ ) lead to an underestimation of magnetic intensities by a factor of 2 to 4 outside of sunspots.

Staude (1969) has demonstrated that the discrepancies between the theoretical and experimental calibration curves may be explained by unresolved horizontal inhomogeneities in the magnetic field region and a similar problem has also been studied by Wittmann. A filamentary model of the magnetic field and magnetic microturbulence with optically thin magnetic turbulent elements have been studied by Stenflo (1968a), who shows that pure microturbulence does not falsify the observed value of the average field, but inhomogeneous nature of the magnetic field may make the measured values too low. 


\subsection{Observations from space vehicles}

(by E. v. P. Smith, University of Maryland)

Probably the most important advance of the last few years has been the increased spatial and spectral resolution of observations made both from satellites and from rockets. For example, Vaiana et al. (1968) have obtained remarkable photographs of flares in the soft X-ray region with a resolution of $1^{\prime \prime}$ during rocket flights, with instrumentation relying on image forming grazingincidence optics. A spectral resolution ranging from $0.01 \AA$ at $2 \AA$ to $0.6 \AA$ at $300 \AA$ was obtained by the Goddard Space Flight Center spectrometer flown on OSO 3 (Neupert et al., 1969). This instrumentation included both crystal spectrometers and a grating spectrometer. OSO 4 included the spectrometer experiments from NRL which covered the wavelength range from 0.5 to $8.5 \AA$. The instrument integrates over the entire solar disk but the wavelength determinations have an error of only about $0.03 \AA$ (Meekins et al., 1968). The Harvard Observatory photoelectric spectrometer experiments have been flown both on OSO 4 and OSO 6 . OSO 4 included the wavelength region 300 to $1400 \AA$ with a spatial resolution of $1^{\prime}$ while on OSO 6 the wavelength range is from 280 to $1360 \AA$ and a resolution of $35^{\prime \prime}$. The instrument has the capability of several modes of operation: Spectroheliograms of the entire disk of the Sun in any pre-selected line in this wavelength range can be made every $8 \mathrm{~min}$, and alternatively in the time scan mode, spectroheliograms $7.0^{\prime}$ by $7.5^{\prime}$ are possible every $30 \mathrm{~s}$. The third mode records spectra at any position within $23^{\prime}$ of the center of the solar disk. In addition to the Harvard spectroheliometer, other solar experiments on OSO 6 include the NRL X-ray spectrometer extending from 0.14 to $25 \AA$, the hard X-ray detector for five energy bands between 20 to $200 \mathrm{keV}$ of the University of Bologna, and the University of London EUV monitor $(180 \AA$ to $L \alpha)$.

Excellent time resolution was attained in the soft X-ray region by the Leicester experiment on OSO 5 which includes a grazing incidence telescope that maps the Sun in 7 bands between 8 and $15 \AA$ with a spatial resolution of $1.6^{\prime}$ and time resolution of $5 \mathrm{~s}$. A second Leicester experiment on the same satellite scans the Sun with an aperture of $2^{\prime \prime}$ by $2^{\circ}$ in the $3-9 \AA$ band every $7 \mathrm{~s}$ (Evans and Pounds, 1968). Leicester is also preparing a new crystal spectrometer to be flown, initially on rockets, which will use field collimators to select and limit the area of the solar disk to be observed rather than integrating over the entire disk of the Sun as in the past. At the Physical Institute of the Soviet Academy of Sciences the group of Mandelshtam, Zhitnik and Tindo has flown X-ray experiments on three satellites of the COSMOS series during 1967-1969 and on the satellite Intercosmos 1. These experiments include $X$-ray photometers for the energy range 2-50 keV and spectroheliograms yielding X-ray spectroheliograms within 2-5 keV with resolving power of about 1'. The Astrophysics Research Unit at Culham Laboratory has flown in a rocket and will fly again in collaboration with the Stockholm Observatory an echelle spectrograph for the wavelength range $\lambda 1200-2200 \AA$. This group is also planning to fly again the two EUV and soft $\mathrm{X}$-ray grazing incidence spectrographs $(\lambda \lambda 15-120 \AA$ and $450-800 \AA)$.

Dijkstra at the Space Research Laboratory at Utrecht is developing fresnel-type zone plates for imaging monochromatically at X-ray wavelengths. One fresnel system has already flown in a NASA rocket in October 1967, and improved instruments are expected to be flown in ESRO rockets in 1970 and 1971. Van Beek and Van Gils of the same group are also developing an equipment for monitoring hard X-ray bursts between 20 and $600 \mathrm{keV}$ in 12 channels, aboard the ESROTD 1 satellite in 1972. Prior to that time balloon flights will test the instrument. Other X-ray spectral observations being planned are those of the group at Paris University under the leadership of Michard, which will include spectroscopic observations from rockets in the spectral range 1.8 to $9 \AA$ and 40 to $80 \AA$, with 2 to $3^{\prime}$ spatial resolution. The first flights are anticipated toward the end of 1970. Valníček's group at the Ondřejov Observatory has flown equipment monitoring solar $\mathrm{X}$-rays in three channels, one of them being for energies $>50 \mathrm{keV}$, on the Soviet satellite Intercosmos 1 in September 1969, and the experiment is planned to be repeated in 1970. In the past, hard X-rays have been observed from OSO 3 by the University of California group under the leadership of Peterson, and on OGO 3 by the Goddard group (Cline et al., 1968). 
The Apollo telescope mount (ATM) for 1972 will carry five solar oriented experiments. These will include ultraviolet spectrographs and spectroheliographs, and X-ray imaging telescope, and a remote occulting telescope for white-light coronagraph. The participating institutions are Harvard Observatory, Naval Research Laboratory, American Science and Engineering, Goddard Space Flight Center and the High Altitude Observatory. The California Institute of Technology, The Jet Propulsion Laboratory and Mt Wilson and Palomar Observatories have participated in the development of a $65-\mathrm{cm}$ solar telescope to be flown on a satellite to obtain extremely high resolution films in various wavelengths. The American Science and Engineering imaging X-ray telescope and a scaled-down version of the Naval Research Laboratory ATM spectroheliograph will be flown on a rocket as part of a flare-watch program late in 1969. Similarly Novick at Columbia is preparing a flare rocket for determining the polarization of flares in the soft X-ray region for 1970. A balloonborne $30-\mathrm{cm}$ telescope with a compact high resolution spectrograph has been developed by Fraunhofer Institute (Freiburg). With a resolution of 0.4 and $11 \mathrm{~m} \AA$ and a guiding stability of 0.1 simultaneous observations of spectra and white-light or $\mathrm{H} \alpha$ pictures are aimed for. The first results of testing of a solar stratoscope were described by Krat (1968). A "solar" space probe going up to 0.3 A.U. is under consideration in cooperation of GFR and USA (Porsche, 1968).

Both Michigan Observatory and Goddard Space Flight Center have flown radio low-frequency dynamic spectrographs to study solar bursts. The Michigan instrument flown on OGO 3 has the range from 2 to $4 \mathrm{MHz}$ while the Goddard instrument has a range from 0.45 to $3.0 \mathrm{MHz}$.

Narrow band filters for optical wavelengths suitable for prolonged satellite operation are being developed by Harvard Observatory in conjunction with Perkin-Elmer Corporation and Thin Film Industries. These are solid etalon Fabry-Pérot interferometers combined with multilayer dielectric filters, with high stability over an extended period. Filters with $\frac{1}{2} \AA$ bandwidth have been made, and wavelengths include $\lambda \lambda 6563(\mathrm{H} \alpha), 6103$ (CaI), 5250 (FeI), 3933 (CaII), and $3968 \AA$ (CaII).

\section{CYCLIC VARIATIONS OF SOLAR ACTIVITY}

(based on a draft prepared by J. Xanthakis, Athens)

From a study of the annual values of the $5303 \AA$ coronal line intensity during 1954-1965 Gnevyshev and Chistjakov (1968) have confirmed the existence of two activity maxima in the 11-year solar cycle. The application of autocorrelation methods by Vitinskij (1967) and Vitinskij and Kopecký (1968) has shown the existence of possible periodic variations of the mean importance of spot groups with periods smaller than 11 years, and in some cases the presence of Gnevyshev's two peaks in the 11-year cycle. Xanthakis' (1969) analysis has shown that these two maxima also appear in the distribution of proton flares and as a function of the spot areas index. Kopecký and Kuklin (1969a) have concluded that the double maximum of the 11-year cycle is determined by the importance of spot groups and not by the frequency of the spot group formation.

Gleissberg (1967) has studied the mean curves of the 80-year solar cycle, which generally show a symmetry of the ascending and descending branch. Zhukov and Muzalevskij (1969), however, have carried out a correlational-spectral analysis of the solar activity periodicities which casts a serious doubt on the reality of the 80-year solar cycle. Their analysis has detected a cycle with a period of about $200 \mathrm{yr}$, with power of the harmonic exceeding powers of all the other long-period components. Vitinskij (1969b) has found that a secular cycle of sunspots, unlike the 11-year cycles, is of the character of free pseudo-harmonic oscillations. An increasing attention has been paid to the hypothetical influence exerted by planets upon the Sun (Memeth, 1967; Bigg and Mulhall, 1967; Takahashi, 1968; Blizard, 1969).

Leighton (1969) has presented a kinematical model of the solar cycle, based upon field amplification by the solar differential rotation. The rather broad quantitative agreement of his model with the known features of the solar cycle led him to a conclusion that other factors which have been suggested as contributing to the solar cycle, such as meridional circulatory currents and torsional oscillations, are unlikely to be important. This model is now being extended to include variation of field strength and sunspot eruption with longitude as well as latitude in the hope of 
elucidating the well-known spatial correlations in sunspot eruptions. At Munich Observatory, Born and Wellmann have derived the diffusion coefficient for magnetic fields from study of the development of CaII plages, which is in good agreement with Leighton's theory. Other models of the solar cycle have been proposed by Nakagawa and Swarztrauber (1969), who have solved the hydromagnetic equations for a differentially rotating Sun and found that the solutions obtained show several interesting topological features of the general magnetic field, and by Steenbeck and Krause (1969) who presented models of alternating field dynamos, which are in good agreement with the real situation on the Sun.

\section{ACTIVE REGIONS ON THE SUN}

(by K. O. Kiepenheuer, Freiburg)

At the 1967 IAU Symposium Structure and Development of Solar Active Regions (Kiepenheuer, 1968 a) a number of important results have been presented. Important contributions also came from the CSSAR Project, organized by Michard (1968), and the PFP Project, organized by Švestka (1968b). The synoptic presentation of solar magnetic fields for the period August 1959 to June 1966 prepared by Howard et al. (1967) represents a basic and indispensable contribution for future investigations of the solar magnetic cycle and the production and evolution of active regions. Also the magnetic classification of active regions, introduced by Smith and Howard (1968) and by Martres (1968), has become an efficient tool in this context.

In this section we shall understand active regions as physical units and not as an assembly of single phenomena. From this point of view, the concept of an active region has undergone considerable changes during the last years. New types of observations as well as the systematic presentation and digital evaluation of solar magnetic data have brought a wealth of new and significant information. The mechanism of an active region is intimately connected with the model of the solar activity cycle. It becomes increasingly clear, however, that the well-known model proposed by Babcock, which is able to explain a number of characteristic features of the cycle quite adequately and which represents, because of its perspicuity and clearness, an enormous stimulus for research, is no more in position to account for these complex observations (Kiepenheuer, 1968b; Bumba et al., 1968). Therefore, it appears most important to work out a consistent global mechanism for solar activity as, e.g., Leighton (cf. Section 3) is attempting to do in his recent theory.

An active region can no more be regarded as an independent organism produced only by general field amplification which is brought to the surface by magnetic buoyancy. The recent studies have shown that the significant factors influencing the birth and the evolution of active regions seem to be mainly the following:

(a) Deep-seated magnetic subsurface sources - probably at the base of the convection layer which produce active regions over a longitude zone of several tens of degrees. These sources rotate with a synodic period of about 27.0 days (Bumba and Howard, 1969; Bumba et al., 1969). These long-lived components in the solar magnetic field distribution, which are obscured by rapidly changing structures of smaller dimensions, are clearly connected with the interplanetary magnetic field structure (Wilcox and Howard, 1969; Schatten et al., 1968) and with the so-called "Bartels Active Longitudes" (Bumba and Howard, 1969). This also seems to have some bearing on the concentration of faculae in longitude (Dodson and Hedeman, 1968). The effect of active longitudes is found most pronounced for the most active phenomena (Levickij, 1968; Vitinskij, 1969; Švestka, 1969), including the proton-flare regions on the Sun (Sakurai, 1967a; Švestka, 1968a; Haurwitz, 1968; Bumba and Obridko, 1969).

(b) Continuous interaction of old background fields with the field of new active regions, the former being responsible for the observed recurrence of active regions. The locations of the centers of recurrence rotate with the 27.0 day period (Bumba and Howard, 1969).

(c) Active regions, once formed usually do not rotate any more with the 27.0 day period. They somehow withstand, however, the shearing effects of differential rotation as long as the magnetic fields involved are strong enough (Bumba and Howard, 1969; Dodson and Hedeman, 1968). 
(d) The formation of a significant number of active regions was followed in numerous instances within a solar rotation by the development of another active region on the opposite site of the Sun, about $180^{\circ}$ away (Dodson and Hedeman, 1968a).

(e) The concentration, dilution and redistribution of magnetic fields is done by a system of convective motions occurring in the convection layer of the Sun: granulation, supergranulation (Bumba, 1967) and the "giant cells", all three interacting with differential rotation of the Sun in a not yet quite perceptible way (Schmidt, 1969).

The concept of giant cells has received support from theory: Simon and Weiss (1968) have shown that the observed concentration of magnetic field between supergranules can be produced only by subphotospheric convection in cells of about $30000 \mathrm{~km}$, which must extend over several scale heights. From such a model a third characteristic scale of motion follows with cells of about $300000 \mathrm{~km}$ in diameter.

\section{FINE-SCALE MAGNETIC FIELDS}

(by V. Bumba, Ondřejov)

Spectrograms obtained during moments of good seeing, making use of the $80-\mathrm{cm}$ solar image at Kitt Peak, demonstrate magnetic fields of several hundred gauss in tiny areas as small as $500 \mathrm{~km}$ in extent in regions sometimes well removed from areas of sunspot activity. They can be observed as weakenings or gaps in temperature-sensitive lines and as local darkening in the continuum (Sheeley, 1967) and are similar to Beckers and Schröter's (1968a) magnetic knots (cf. subsection 7.2). Time-lapse sequences of spectroheliograms using Leighton's photographic technique allowed to be found a steady flow of these tiny field concentrations laterally outward of sunspots and the birth of new elements of this network (Sheeley, 1969). Such photographic observations seem to attack one of the most important problems of the dynamics of active region development. On the other hand, however, line scans with a photoelectric magnetograph using the same instrument did not show magnetic elements below about $1500 \mathrm{~km}$ in size (Livingston, 1968). The influence of the resolving power of a magnetograph on statistical results concerning the mean field strength, the average size and the number of magnetic elements of different polarity was examined by Severnyj (1968a).

The reality of tiny concentrations of magnetic lines of force and their relations to flares and bright points has been confirmed by the photographic technique using a birefringent filter (Ramsey et al., 1968). The importance of such small-scale fields in the field diffusion was considered theoretically by Kassinskij (1969) who showed that these fine-scale field elements are transported through the large-scale motions during a very long time without any considerable damping.

At Capri (Deubner, 1968) and Crimea (Severnyj, 1968c) short periodic oscillations, with periods from 7 to $9 \mathrm{~min}$, of magnetic field strength have been found on repeated scans along a fixed line, or on one place on the disk, outside of active regions. These measurements were carried out photoelectrically with relatively high resolution, the aperture slit of the magnetograph being of the order of 10 square seconds of arc. At Capri close correlation has been found between these oscillations and simultaneously measured contrast changes. The physical nature of this phenomenon is unknown, although the seeing cannot yet be fully excluded as its possible source.

\section{FACULAE AND PLAGES}

(extracted from a draft* prepared by G. Godoli, Catania)

Daily maps of $\mathrm{H} \alpha$ and $\mathrm{K}$ plages observed at Catania have been made since 1967, and characteristic figures are regularly published in Osservatorio Astrofisico di Catania Pubblicazioni. The McMath-Hulbert Observatory has continued its daily measurement of position, area, and intensity of $\mathrm{K}$ plages and has distributed these measurements through the World Data Center A, ESSA, Boulder. Regular observations of faculae and plages also have been made at Kislovodsk. Definitive

* Available on request. 
characteristic figures of $\mathrm{K}$ plages for IGY and IQSY and maps of $\mathrm{K}$ plages for the second year of IQSY were published by Godoli (1967) and Godoli et al. (1967). Starting January 1968 the Rome Observatory regularly publishes films with reproductions of daily $\mathrm{H} \alpha$ and $\mathrm{K}_{232}$ images of the Sun as The Photographic Journal of the Sun.

Loughhead et al. (1968) have shown that chromospheric faculae regions seen in $\mathrm{H} \alpha$ may be partially resolved into a granular structure. According to Bray (1968) the correlation between photospheric faculae and plages is weaker than has hitherto been supposed. A detailed study on the chromospheric and photospheric network is in progress at Kitt Peak, carried out by Chapman, Engvold and Sheeley. Spectroheliograms obtained by Fredga (1969) in the Mg II $\lambda 2795.5 \AA$ line have been found very similar to $\mathrm{K}$ spectroheliograms, and a detailed comparison between $\mathrm{MgII}$ and $\mathrm{Ca}$ II is in progress.

A correlation has been found between the $\mathrm{K}$ plage intensity and the $L a$ (Monsignori Fossi et al., 1968), and the X-ray flux (Landini et al., 1968), respectively. A correlation between enhanced radio emission at $9.1 \mathrm{~cm}$ and X-rays found by Reidy et al. (1968) indicated a common physical origin of both these types of radiation in the plage region.

The newly constructed double-beam-splitter magnetograph at Kitt Peak is currently used by Sheeley to study the relation between intensity and magnetic fields of plages. Magnetic fields in plage areas have also been discussed by Tandberg-Hanssen, Porter, Bumba and Godoli at the IAU Symposium no 35, as well as by Steshenko (1968) and Livingston (1968). Photospheric and chromospheric longitudinal magnetic fields and the brightness of plages have been compared by Tsap (1967), who also studied the correlation between longitudinal and transversal magnetic fields on different heights (Tsap, 1969). The correlation between velocities at different levels has been investigated by Gopasjuk and Tsap (1969b). Gopasjuk (1967) has shown that the brightest portions of a plage as well as the brightest points of the chromospheric network coincide with the neutral line of the radial velocity or lie close to it. The gases move both toward and from the neutral line and do not correspond to the pattern of supergranulation (Gopasjuk and Tsap, 1969a).

At IZMIRAN in Moscow, Ioshpa, Mogilevskij, Obridko and Shelting are using simultaneous measurements at two different levels in the photosphere and chromosphere for constructing the spatial structure of the magnetic field for the whole active region, and for this purpose active prominences have also been used by Ioshpa. Mogilevskij and Shelting also use simultaneous magnetic and velocity observations in order to reveal three-dimensional interconnection of the largescale elements in the magnetic and velocity fields. Alexandrovich and Stepanov (1969) have detected regions of compressed and rarefied plasma in the chromosphere, and found that the brightest parts of plages are situated in the regions of compressed gas.

Weart and Zirin (1969) have shown some characteristic features, that occur in plages prior to sunspot occurrences, some of them confirming discoveries made three decades ago by Waldmeier. Other work of these authors is in progress. They find an unequal development of the $\mathrm{H} \alpha$ plage brightness in association with the preceding and following part of the active region, respectively, while this brightness asymmetry does not appear in the K-line. They also have attempted to determine weak magnetic fields by examination of on-band and off-band high-resolution $\mathrm{H} \alpha$ pictures and report that $5-\mathrm{G}$ contours of plages can be distinguished in this way. At the Cambridge solar magnetograph operating in Malta, observations of weak magnetic fields have been carried out since early 1966 (Von Klüber, 1968), which may contribute to the understanding of plage formation. At McMath-Hulbert Observatory Mohler is investigating the correlation between magnetic measurements and line profiles in plages.

Heights of plages have been studied by Öhman at Kitt Peak comparing $K_{2}$ and $K_{3}$ components in limb phenomena and work of this type is now under consideration at the Swedish station at Anacapri. Structure of plages has been discussed by Anichini and Godoli (1967), Livshitz (1968) and Bachmann (1969). Zirker (1967) has found larger velocities in a plage than reported for spicules in quiet regions. Sitnik and Mitropolskaja (1968) have concluded that turbulent velocities in faculae exceed the photospheric values and the same result is obtained by Bodajan and Livshitz. Current investigations carried out by Mitropolskaja and Sitnik show that the turbulent velocities decrease 
with the depth. According to Howard et al. (1968) the 5-min oscillations are damped in plages relative to the quiet photosphere, which also has been confirmed by E. Smith (1969). Mazzucconi and A. Righini are studying area and intensity fluctuations of plages in order to establish whether these fluctuations are preferentially present before or after flares, and photometric investigations on the flare-associated plages are in progress at the Kandilli Observatory by Soytürk.

At Harestua Observatory observations of photospheric faculae in several wavelength regions are at present carried out. From photoelectric measurements Schmahl (1967) has found that the intensity differences facula/photosphere are non-zero even for weak faculae in the disk-centre, and therefore it is not necessary to suppose a temperature deficit in deep layers of faculae. In the infrared, the facula contrast has been studied by Kuzminykh and Sitnik (1967). A comparative study of hydrogen lines in spectra of faculae and photosphere has been carried out by Vojhanskaja (1967). Pande et al. (1969) have calculated the dependence of facula/photosphere contrast in molecular lines on dissociation energy and the CO molecule in faculae has been studied by Polonskij (1967).

Some other papers on the structure and development of plages can be found in IAU Symposium no. 35 and Annals of IQSY, Vol. 3.

\section{SUNSPOTS}

(by W. Mattig, Freiburg)

\subsection{Sunspot spectrum and abundances}

For the last thirty years the Utrecht photometric atlas of the solar spectrum was extremely helpful for the whole solar research. Now two similar publications for sunspots are in preparation. At the Kitt Peak Observatory maps are made for a photographic atlas of the umbral spectrum taken through a Babinet compensator, and photochromic glass is utilized to reduce the photospheric to umbra intensity gradient. At the Locarno station of the Göttingen Observatory photoelectric records have been made by Schröter and Wöhl in the wavelength region 3900-8000 $\AA$ in order to produce a photometric atlas of an umbra spectrum.

The abundance of some elements has been determined from high dispersion spot spectra. Li, In, $\mathrm{Rb}$ and $\mathrm{Cs}$ have been studied by Wiehr et al. (1968). A special program to determine the lithium abundance very accurately from the $6707 \AA$ line is under way by Beckers and Hoyt. The appearance of the elements $\mathrm{Eu}$ and $\mathrm{La}$ in sunspots has been investigated by Bachmann, Pflug and Staude at Potsdam. Kumar at the McMath-Hulbert Observatory and Branch (1970) have determined the abundance ratio of $\mathrm{Mg}$ isotopes, which significantly differ from the terrestrial values.

\subsection{Sunspot fine structure}

A substantial progress has been made in the field of the fine structure in spots and their surroundings. Beckers and Schröter (1968b) who observed the umbral fine structure (umbral dots) in two continuum wavelengths, came to the conclusion that, after correcting their measurements for the influence of finite resolution, the umbral dot's intensity is nearly the same as the photosphere, and that the dots have a true diameter of 150 to $200 \mathrm{~km}$. These umbral dots are concentrated mostly in the outer part of the umbra and have a lifetime of $\sim 25 \mathrm{~min}$. Wilson (1969b) has discussed several models with these bright structures on the basis of a three-dimensional radiative transfer analysis.

A very rapidly changing fine structure in the umbral chromosphere was detected by Beckers and Tallant (1969) and confirmed by Wittmann (1969). These bright features, observable in the K-line by filters and with the spectrograph, and called umbral flashes, have diameters smaller than $2700 \mathrm{~km}$, magnetic field strength of about $2000 \mathrm{G}$, and there are usually about 3-5 flashes in an umbra at the same time. The life-time of such a flash is about $50 \mathrm{~s}$ and there is a tendency for their repetition after about $150 \mathrm{~s}$. The umbral flashes show a proper motion of $40 \mathrm{~km} / \mathrm{s}$ in the penumbra direction and Doppler velocities of $6 \mathrm{~km} / \mathrm{s}$. Havnes in Oslo has examined the hypothesis that an umbral flash may 
be caused by a magnetoacoustic wave phenomenon and, in general, the observations agree with the wave hypothesis.

The chromospheric fine structures in $\mathrm{H} \alpha$ in the surroundings of spots have been studied by Loughhead (1968). The dark filaments, fibrils, were found to be the continuation of the filamentary structure of the penumbra. In the case of this "superpenumbra", most fibrils end within the penumbra and some penetrate right to the border of the umbra. The fine structure of the intensity variation and magnetic field around sunspots has been investigated by Beckers and Schröter (1968a). The so-called magnetic knots coincide with dark intergranular space. More than 2000 knots can be found around a unipolar spot. The diameter of a typical knot is about $1100 \mathrm{~km}$, the magnetic field amounts up to $1400 \mathrm{G}$, it generally shows a downward motion and has a iifetime of more than $30 \mathrm{~min}$. It seems that there exists a critical level of the magnetic field $(\sim 1500 \mathrm{G})$ for the smallest sunspot to appear (Bumba, 1967b; Steshenko, 1967). Similar to the magnetic knots are Sheeley's (1967) "gaps" (cf. Section 5). Both knots and gaps coincide with regions of bright CaII emission. An attempt for an explanation of these phenomena has been presented by Zwaan (1967).

\subsection{Sunspot magnetic fields and motions}

The magnetic field distribution over an isolated spot has been investigated again by Beckers and Schröter $(1969 b)$. The distribution of the total magnetic field across a spot is given by $H(r)=$ $=H(0)(1+r / R)^{-1}$ and the inclination of the field vector is nearly the same as found by Nicholson. Deubner and Göhring at Freiburg are in agreement concerning the inclination angle, but the field distribution differs remarkably. They found a lower field strength in the outer part of the spot. Kjeldseth Moe (1968b) has found a field distribution in good agreement with that of Bumba of 1962. As Beckers and Schröter have confirmed, the magnetic field does not drop to zero at the boundary penumbra-photosphere, which is in accordance with Kassinskij's (1967) remark that the magnetic radius of a spot is larger than that of the penumbra. Recordings of the magnetic field at different photospheric levels made by Kotov (1969) show the presence of a strong transversal field within the umbra and large (up to $90^{\circ}$ ) changes in the orientation of $\mathrm{H}$ with depth in certain parts of the spot. He also obtained the distribution of the vertical and horizontal components of the electric current $\left(\sim 10^{3}-10^{4}\right.$ CGSE).

The time variation of the maximum magnetic field strength observed by Kolpakov (1968) shows a wide range of $10^{-4}-5 \times 10^{-2} \mathrm{G} / \mathrm{s}$ for different spot types. Ikhsanov (1967) has found that the magnetic field strength varies with time in parallel to the change of spot area, but the rate of change is smaller for larger spots. Jakimiec is investigating the distribution of the magnetic force and electric currents in the surface layers of sunspots. Künzel (1969) has demonstrated that the shape of the penumbra is very closely correlated with the distribution of the magnetic field polarity in sunspot groups, which means that the shape of the penumbra is influenced by the magnetic field of spots in the neighbourhood.

The fine structure of the magnetic field in the penumbra has been studied by Beckers and Schröter $(1969 a)$ and by Mattig and Mehltretter (1968). The results are contradictory: Beckers and Schröter find the stronger and more horizontal magnetic field in the interfilamentary dark regions, while the result given by Mattig and Mehltretter is vice versa. In accordance to each other are the results on the velocity fine structure by Beckers (1969) and Mattig and Mehltretter. The Evershed effect is restricted to the dark interfilamentary regions in the penumbra. Steshenko and Shcherbakov (1969) have discovered motions of the type of pulsations in the radial direction, along the penumbra filaments. Obviously, more observations are needed to determine the physical parameters in the penumbral fine structure elements.

At Oslo, Maltby and Bönes have made observations to study the Evershed velocity fine structure. Haugen's (1969) studies of the chromospheric velocity field have revealed that the velocities of different elements vary in a wide range of -35 to $+50 \mathrm{~km} / \mathrm{s}$. The mean velocity vector is directed towards the spot and downward, the inclination being about $20^{\circ}$ and the mean velocity amounts to $7 \mathrm{~km} / \mathrm{s}$, but the rms deviation of line-of-sight velocity is of the same order. The depth gradient of 
line-of-sight velocity near the penumbral radius has been determined by Sattarov (1969) as about $0.01 \mathrm{~km} / \mathrm{sec} / \mathrm{km}$. Chitre (1968) has discussed the Evershed flow from a general point of view. Assuming that the Evershed flow starts from the base of the spot with a velocity that is adequate to carry the convective flux at that level, the velocity at the surface turns out to be of the order of $1 \mathrm{~km} / \mathrm{s}$, in agreement with the observations.

Strong efforts have been made to explain the measured polarization and line contours of magnetically split lines by solution of the transfer equations of the Stokes parameters under different conditions, as for example by Rachkovskij (1967a) and Kjeldseth Moe (1968a), who has obtained very complete solution of the equations with a homogeneous field but without any special assumption regarding the model atmosphere. Kai (1968b), Beckers (1969) and Göhring have looked for the influence of the Faraday rotation. Numerical integrations of the transfer equations of the four Stokes parameters have been carried out by Kjeldseth Moe, Beckers (1969), and Göhring to investigate the accuracy of the simple Unno theory and the influence of the curvature of the magnetic field. Stenflo (1969b) has shown that the reradiated light in the case of longitudinal Zeeman effect will not be completely circularly polarized, if the life time of an atom in an excited state is comparable or shorter than the period of Larmor precession. Most recently, the problem of line formation in a magnetic field has also been discussed by Rees (1969).

An interesting discussion has developed around the problem of the appearance of a $\pi$-component of a normal Zeeman triplet in sunspot umbrae, which many a time shows circular polarization opposite to that of the main Zeeman splitting. Recently, e.g., Deubner (1969) has measured in the " $\pi$-component"an opposite circular polarization corresponding to a magnetic field of $600 \mathrm{G}$. Henoux (1968a) has interpreted the existence of this $\pi$-component as a saturation effect and Göhring (1969), applying Unno's theory of line formation in a magnetic field for the case of true absorption arrived at the same conclusion. But, as Beckers and Schröter (1969a) have shown, the Stokes parameter $V$ cannot be explained by the saturation effect described above. Possibly magnetic field inhomogeneities caused by umbral dots are responsible for the existence of the $\pi$-component. This is in agreement with the results of Mehltretter (1969) who inspected weak and medium strong magnetically affected lines. Interesting observational results to the solution of this problem have been added by Surkov (1969) who demonstrated that not only the presence and behaviour of the $\pi$-component, but also the asymmetry of the whole split line, depends on the age, size, and heliographic position of the studied sunspot.

\subsection{Physical state of sunspots}

A review paper on the structure of sunspots has been written by Zwaan (1968). Many observations of the sunspot spectrum have been made and lots of results are available to derive umbral and penumbral models. According to Rossbach's observations at Göttingen, the spot intensity is nearly independent of the area, even for spots or pores with diameter down to 2". Henoux (1968b) has also assumed that all spots have the same intensity. These results are in contradiction to measurements carried out by Kneer and Mattig (1968). From far infrared observations ( $\lambda 24 \mu$ ) Léna (1969) has derived umbral temperatures near $3000 \mathrm{~K}$ and penumbral temperatures of about $4000 \mathrm{~K}$. At the Oslo Observatory several pinhole photometers have been used to determine the umbral intensities in a wide wavelength region $4040 \AA<\lambda<40 \mu$. A very small temperature gradient in the umbra at $0 \cdot 2<\tau_{0}<1$ was called for by Mattig (1969a) from the reduction of older limb darkening observations, and by Wittmann and Schröter (1969) from new observations. Wilson (1969a) has demonstrated that a low temperature gradient is a consequence of the influence of umbral inhomogeneities on the center-to-limb variation.

Teplitskaja and Turclina (1969) have discussed once more the equivalent width measurement by Elsässer and Fricke and came to the result that the lines are formed in a dense sunspot with a hydrostatic pressure distribution. Ruhm's (1969) rediscussion, however, establishes the former results of Fricke and Elsässer. For two different sunspots Henoux $(1968 a, b)$ has derived homogeneous sunspot models for the depth range $10^{-5}<\tau_{0}<8$. His results include umbra in hydrostatic equilibrium, transparency of the spot, microturbulent velocity equal to that of the photosphere, and 
no radiative equilibrium in layers deeper than $\tau_{0}=1$. Obridko $(1968 b)$ has shown that the magnetic field determined from ionized lines is only half of that measured in neutral lines and proposed a two component sunspot model, with $5-10 \%$ bright elements covering the umbra.

Elste at McMath-Hulbert Observatory has obtained high dispersion spot spectra, which provide the basis for a number of current research projects. Hagyard and Harvey at Kitt Peak have employed photoelectric and photographic techniques for a comparison of theory and observation of the line profile of FeI $\lambda 5250$ in sunspots. Wiehr at Göttingen and Kneer at Freiburg are using magnetically unaffected lines to establish sunspot models. At Utrecht, first preliminary results by Zwaan show a microturbulence velocity smaller than $1 \mathrm{~km} / \mathrm{s}$ for non-split lines and a temperature gradient somewhat steeper at $\tau_{0}>3$ than that of Henoux. As molecular lines are concerned, Makita (1968) obtained a rotational temperature from TiI lines of $3000 \mathrm{~K}$ while Webber (1969) found remarkably higher temperatures using $\mathrm{MgH}(3900 \mathrm{~K})$ and $\mathrm{CaH}(3500 \mathrm{~K})$, respectively. Measurements of molecular line strengths have been made by Sotirovski at Meudon and by Wöhl (1969), who could identify $\mathrm{H}_{2} \mathrm{O}$ and $\mathrm{NiH}$ in sunspots.

Kjeldseth Moe and Maltby (1969) have derived a homogeneous penumbra model in hydrostatic equilibrium which is able to explain the observations of the continuum and the equivalent widths. From highly resolved white light photographs and spectra Beckers, Schröter and Wittman have determined intensity fluctuations, curves of growth and line wings of strong lines. The aim is to deduce an either homogeneous or inhomogeneous model of the penumbra.

The height of formation of several lines was determined by Mattig (1969) from sunspot observations very close to the limb. He deduced the same scale height in the umbra as in the photosphere and therefore hydrostatic equilibrium. Ruhm (1969) has rediscussed these observations and found a remarkably larger height-scale in the umbra. Engvold (1969) has found height differences between the $\mathrm{H} \alpha$ core and the continuum in the range 1000 to $2300 \mathrm{~km}$. From the displacement of the sunspot border in the lines of FeI and Tir Baranovskij (1969) has found that the umbra is 10 times more transparent than the photosphere, and the displacement of the $\mathrm{H} \alpha$ line leads to the height of formation of this line equal to $740 \mathrm{~km}$ above the photosphere. He has also found $P_{\mathrm{g}}$ and $P_{\mathrm{e}}$ in the umbra to be 1.5 to 2 times lower, and the gradient of gas pressure somewhat higher than that computed for the undisturbed photosphere. Hence, one is induced to conclude that the pressure and height scales are indeed different from spot to spot. There are probably spots in hydrostatic equilibrium and other spots which differ remarkably from this condition.

The Wilson effect has been studied by Wittman and Schröter (1969), who derived an umbral depression of about $650 \mathrm{~km}$ and a density scale height of about $450 \mathrm{~km}$, in good agreement with the results given by Mattig (1969b). By analyzing the three-dimensional equation of radiative transfer, Wilson (1968b) has shown that by assuming a spot depression of $700 \mathrm{~km}$ near unit optical depth it is possible to reproduce qualitatively all features of the observed intensity profiles. According to Jensen et al. (1969), hydrostatic models for the penumbra and umbra and a depression of $600 \mathrm{~km}$ give a good fit for both the Wilson effect and the intensity profiles near the limb.

\subsection{Theory}

Erikson and Maltby (1967) have suggested longitudinal compression waves propagating parallel to the solar surface from the umbra to the penumbra, and could show that the resulting line profile is asymmetrical in the same sense as observed in the penumbra. With the Evershed effect interpreted as an acoustical wave phenomenon, the propagation may also be perpendicular to the magnetic lines of force. The effect of magnetosonic waves on special lines was considered by Maltby (1968a) with the result that magnetically active lines show a larger wavelength shift than inactive lines. He believes to have found this effect in the Evershed observations by Servajean. Maltby $(1968 b)$ has also shown that the effect of Alfvén waves, in terms of line broadening, should be largest in regions where the magnetic field is perpendicular to the line of sight.

Deinzer's sunspot theory has been improved by Yun (1968), introducing new boundary conditions. A refinement of an earlier sunspot model has been given by Chitre and Shaviv (1967). Wilson 
(1968a) has brought up new aspects concerning the structure and development of a sunspot. He starts with an inhibition of the convection by the magnetic field in the deep layers, resulting in the formation of a "cool zone" above that region, and the development of the penumbra is described as due to the interaction of strong magnetic fields with thermal forces. Altschuler (1967), taking into consideration that the conductivity gradient near the spot boundary may be relatively large, finds that the photosphere is current-free and that ring currents might surround the sunspots. The development in time of a single sunspot has been described by Altschuler et al. (1968). With the initial condition $v=0$ everywhere and a magnetic field distribution of a ring current embedded in the photosphere, it can be shown that Evershed motion will develop within a wide range of initial parameters assumed. Convective instability and overstability in the presence of a uniform vertical magnetic field has been studied by Saito and Kato (1968). The overstable convection may be a cause of some dynamical features (umbral dots) but cannot be the main contributor to mechanical energy transport. Getling and Tverskij (1967) have described a possible mechanism for producing sunspot magnetic fields.

Kopecký and Obridko (1968) and M. Kopecký (1969) have drawn attention to the fact that Joule dissipation of magnetic fields might play a rather important role in some active processes on the Sun. The dissipation time of the spot magnetic field has been discussed by V. Kopecký (1968). Assuming a number of realistic models for the magnetic field, he shows that the dissipation time comes close to the time determined according to Chandrasekhar's formula. The conductivity coefficient for a sunspot model has been derived by Oster (1968) and a recomputation of the electric conductivity was carried out by Kopecký and Kuklin (1969), within a wide range of temperature and gas pressure. The conductivity, however, becomes lower by several orders of magnitude if the interaction between particles and ionic-sound waves is taken into account, which is an effect currently studied by Kuklin and Ribakov.

\section{SOLAR FLARES}

In 1968, two symposia were held on the subject of solar flares: in Tokyo, on Solar Flares and Space Research, the Proceedings of which contains 13 review papers on various aspects of the flare and flare-associated phenomena (de Jager and Švestka, 1969), and at Anacapri, on Mass Motions in Flares and Related Phenomena (Öhman, 1968). Other review papers on flares were presented by Bruzek at the Conference on Plasma Instabilities in Astrophysics, held at Pacific Grove in October 1968, and by Axford and de Jager at the Third ESLAB/ESRIN Conference held in Noordwijk in September 1969.

When compared with the years preceding the last Draft Report, one observes that after 1966 the main attention has shifted from studies of the common chromospheric flare phenomenon to space investigations of the high-temperature flare regions in the corona, and to flares associated with particle events in space. This obviously has been a natural consequence of the fast progress in space research.

\subsection{Spectrophotometry of flares}

Only relatively small progress has been achieved in spectrophotometry of flares. Isophotal photometry of flares has been carried out at the Kandilli Observatory (Dizer, 1969), and a photometric analysis of high-resolution pictures of flares, photographed at Athens, is being made by Falciani, Macris and Rigutti. A new photographic technique of constructing flare isophotes has been suggested by Falciani et al. (1967), and a special photometric analyzer called "videometer" has been developed by Tallant (1970) and is now being used at the NASA Manned Spacecraft Center in Houston.

Central intensities and equivalent widths of 918 emission spectral lines in a flare spectrum have been published by Polupan (1968). Electron density, determined from Hydrogen Balmer series in 16 flares, has been discussed by Fritzová and Švestka (1967) in dependence on the flare importance, phase of development, and on the height in the solar atmosphere, and spectrum of the flare of September 2, 1966, has been analyzed by Stepanjan (1969) who has confirmed the existence of 
"flare knots" and "interflare matter" in the flare region. Babin (1969) has discussed striking differences in the $\mathrm{H} \alpha$ and $\mathrm{K}_{232}$ emission in some knots of the limb flare of 11 July, 1966. Severnyj $(1968 b)$ has shown that the line asymmetry observed in spectra of flares and moustaches, can be explained by incoherent scattering in contracting and expanding shells, with a gradient of velocity inside. He has also found that in many cases the effect of asymmetry in limb flares is connected with a splitting of the flare emission into two distinct features, separated slightly along the dispersion, as if we had two emitting and partly overlapping layers, moving in opposite directions along the line of sight.

Steshenko (1970) finds that in most cases the continuum emission of flares appears in other regions of the flare than the line emission and supposes that this difference depends mainly on the separation of the emission sources in the horizontal direction. The mechanism for the continuous emission, proposed by him, is a plasma jet moving with a velocity of about $100 \mathrm{~km} / \mathrm{s}$ from the flare, which hits the photosphere and increases its temperature up to $6700 \mathrm{~K}$.

\subsection{Flare association with active regions and magnetic fields}

Zirin and Werner (1967) and Moreton and Severnyj (1968) published detailed analyses of the large spot group of September 13-26, 1963, during its transit over the solar disk. Simultaneous occurrence of flare brightenings in regions of opposite magnetic polarity, mostly very closely adjacent to the line $H_{11}=0$, close association of flares with "bifurcated" regions, and well expressed coincidence of flare knots with the strongest electric currents are among the principal results obtained by Moreton and Severnyj. Zirin and Werner found that most flares in the studied region were homologous and some were triggered by disturbances elsewhere in the region. This seems to be in support of Gold's (1968) opinion that it is unlikely that one simple instability is responsible for the flare event within its whole extent. If, however, due to some disturbance, a limited volume is taken out of the region of stability, the energy release in this volume causes a disturbance, which is responsible for triggering all the other accessible instabilities in the region.

As the homology is concerned, an astonishingly good example of two extensive homologous flares occurring nearly 54 apart, has been presented by White and Janssens (1970). The homology included flare shape, filament agitation and other associated prominence activities, as well as close similarity in the microwave radio bursts. Convincing examples of radio homology were earlier presented by Fokker (1967), and examples of X-ray homology were published by Pintér (1969). On the other hand, McKenna-Lawlor (1968) in her analysis of the active region of 1959, July 7-21, has cast some doubt on the actual "homology" of flares. McKenna's studies of this region are still in progress.

The relations of flares to magnetic fields were reviewed by Severnyj (1969a). Severnyj and Zvereva (1969) have found that the change of the net magnetic flux before flares leads to the e.m.f. $\sim 10^{8}-10^{9} \mathrm{~V}$ in the fixed circuit containing the active region, and for important flares this emf is about 10 times larger than the emf due to inductance as it can be evaluated from the measured velocities. In the case of the proton flare of 1966 July 7, Severnyj (1969b) has found a striking variation of the longitudinal field gradients and total magnetic flux, which dropped from highest values before the flare to low values after its occurrence. The flare appeared in the area of the junction of oppositely directed electric currents (Severnyj and Zvereva, 1969) and the brightest flare portions have been identified with the areas of strongest currents. Smith and Ramsey (1967) have found a strong correlation between the shape and intensity of the $\mathrm{H} \alpha$ fine structure and $H_{\|}$, except in the vicinity of the $H_{\|}=0$ line.

Bruzek (1968) has concluded that changes in the chromospheric fine structure suggest changes in the magnetic field associated with the flare occurrence. Malville and Tandberg-Hanssen (1969) have discovered field fluctuations in the flare of 1967 May 21, with average period $7.5 \mathrm{~min}$ close to the period observed in photospheric fields (cf. Section 5). At Culgoora, the magnetograph designed for moderately high angular resolution and rapid frame repetition rates is being used to look for rapid changes in magnetic fields around times of solar flares. 
According to Fortini and Torelli (1968), the most energetic solar regions are due to a fusion of two or more active centers. Martres (1968) has shown that the magnetically complex active regions, in which the most energetic flares occur, only are born at positions where an old active center has existed before, and the complexity is greater if the old active center is younger. This might explain why the non-random distribution of activity in solar longitude is most pronounced for the most important solar phenomena, and for the proton-active regions, in particular (cf. Section 4). Martres et al. (1968) have also found that flares are often associated with two spotted evolutionary features evolving in opposite senses, one growing, the other declining.

\subsection{Flare motions}

At the Anacapri Nobel Symposium (Öhman, 1968), our rather limited knowledge on motions in flares has been summarized by Švestka (1968c) and Dodson and Hedeman (1968b). Attention has been drawn, in particular, to ascending motions observed at some limb events during the first 2-5 min of their development, which may be missed in the disk flare spectra, and to the expansion of two-ribbon flares. Švestka pointed out the difference between the behaviour of flares of this type when occurring inside sunspot groups and outside of them, and Dodson and Hedeman are now currently studying the properties of well observed, major $\mathrm{H} \alpha$ flares in regions with either very small or no detectable spots. The expansion of two-ribbon flares associated with type IV radio bursts has been studied in detail by Ogir $(1967,1969)$ who finds an increase of the ribbon velocity with the increasing magnetic field gradient, and generally, the flare motions directed along the vectors of the transversal magnetic field. On the other hand, the rate of drift of a separating ribbon has been found increasing with the increasing distance from strong magnetic fields by Smith and Ramsey (1967).

Bruzek's study of fine structure of flares has provided evidence that brightening and expansion of small flare elements are associated with or preceded by enhanced mass motion and fast changes in the surrounding small-scale chromospheric structures and that inflow of material is prevailing. This may be considered as a support for Hyder's (1967) impact theory. A study by Janssens and White (1970), which shows a proximity of the falling material to the flare brightenings, also indicates that an impact-brightening process plays a role in the energy release. At Culgoora, observations of the flare fine structure lead to the conclusion that apparent motions in flares are due not to velocity of the flare material, but to successive brightening of new flare fibrils, which appear to have precisely the same pattern as the pre-existing chromospheric structures in the same region.

\subsection{Proton flares}

Great attention has been paid to the particle-producing flares on the Sun. The results of the first Proton Flare Project (PFP) concerning the July 1966 event have been published in Annals of the $I Q S Y$, Vol. 3. This book contains 60 original contributions on the proton-active region, the proton flare itself, and its consequences in space and in the magnetosphere and ionosphere of the Earth. A summary on the development and activity of the region has been prepared by Banin et al. (1969), on energetic particles by Krimigis (1969), and general summary and conclusions have been written by Simon and Švestka (1969). Solar aspects of the July PFP period have been summarized by Svestka (1968b). Summary on the results of the second August/September 1966 PFP period, has been prepared by Švestka and Simon (1969). Fairly extensive PFP results obtained in GDR were published by Krüger and Lauter (1969).

Something like a retrospective PFP was organized by Virginia Lincoln (1969), who prepared a valuable compilation of data on the solar particle event of May 23, 1967 and its geophysical effects. There are contained, among others, contributions by De Mastus and Stover (also see 1968) on the white-light flare emission, Dodson and Hedeman on the $\mathrm{H} \alpha$ flare, McIntosh on the spot group, and several authors contributed on the radio burst. Van Allen, and Kane and Winckler contributed on the X-ray burst, and Lanzerotti (also see 1969) on the particle event. A similar study is now in progress for other selected periods. Another enterprise of somewhat similar type was the Third 
ESRIN/ESLAB Conference, held at Noordwijk in September 1969, aimed in particular at the cosmic-ray flare of 1969 February 25. A second Proton Flare Project organized by Svestka and Simon from May to July 1969 , unfortunately did not meet with success, due to an unexpectedly low solar activity during that period.

Due to the limited extent of the Draft Report, we cannot review here all the numerous results obtained during the 1966 Proton Flare Project, and the readers are referred to the abovementioned summaries, and to the extensive review paper by Svestka and Simon (1969), in particular. From the solar point of view, perhaps the most valuable results, hardly obtainable without the organized PFP enterprise, concern the changes in the active regions prior to the proton fiare occurrence, which start to occur about three days in advance (Severnyj, 1969b; Friedman and Kreplin, 1969; Tanaka et al., 1969a) and become, at least in some cases, very pronounced during the last $12 \mathrm{~h}$ preceding the flare event (Popovici and Dimitriu, 1969; Dodson, 1969; McIntosh, 1969; Friedman and Kreplin, 1969). A uniquely low elevation condensation in the corona was detected following the proton flares of 1966 July 7 and September 2(Newkirk et al., 1969). Eliseeva (1967) and Křivský and Nestorov (1968) have found that SID effects are associated with substantially more flares in proton-active regions than in other regions on the Sun. An association of high-energy flares with transient umbrae has been suggested by McKenna-Lawlor (1969).

In the flare of 1966 August 28, Zirin and Russo-Lackner (1969) have observed a very fast separation of the two bright ribbons during the initial phase of the flare development, which slowed down after a few minutes. The fast expansion coincided in time with the hard X-ray burst, and this observation confirms the earlier conclusion made by Valnícek (1967) that the fast flare expansion, the first maximum of the microwave radio burst, and the maximum of hard X-ray burst, all coincide in time. One can suspect, therefore, that this is the flare phase when acceleration process takes place in the proton flare region (Svestka, 1969). At the proton flare of 28 August, 1966, also the first evidence of a strong ultraviolet flash was recorded by McClinton and Kreplin, coincident again with the hard X-ray burst. A possible relationship of this UV enhancement to the continuum of the white-light flare has been suggested (Friedman, 1969).

Problems related to short-term forecasts of flares and proton flares, in particular, have been discussed and reviewed by Simon et al. (1969) and Severnyj and Steshenko (1969). The problems associated with long-term forecasts of proton flares have been discussed by Sivestka $(1968,1969)$. Attempts to work out a method of long-term regional forecasts of flares are also made at Pulkovo by Vitinskij and his co-workers. A classification system of solar proton events is being prepared by Smart and Shea.

\section{FLARE THEORIES}

The efforts of theoreticians in trying to find an explanation for the flare process have not diminished during the past three years, and many new or modified theoretical considerations on this problem have appeared. Some of them, including older theories, have been critically reviewed by Sweet (1969).

Unsöld (1968) has proposed that flares should be considered as non-stationary analogues of plages, caused by sudden splashes of the waves that originate in the turbulent convection zone. The produced fast heating in the chromosphere produces shock waves giving rise to the flare-associated high-energetic phenomena. Pneuman (1967) has suggested that flares may be caused by chocking off of the normal energy flux in the corona by the closed magnetic fields of a plage. In this model, the flare is produced by the sudden increase in energy density of trapped kinetic and magnetic energy, which initially might be in the form of Alfvén waves and later could be partitioned into a wide variety of other forms including the acceleration of high-energy particles. Menzel (1968) has tried to show that the flare can be simply due to an existing natural conflict between mechanical and thermodynamical processes, which prevents the development of either a static or a steady-state equilibrium in a sunspot group. He has shown that a dynamic outward flow pumps gas in between the sunspot pair in a bipolar group, thus giving rise to a pressure and temperature increase in that region. After breaking the restraining magnetic barriers the gas explodes and a shock wave develops.

Schatzman (1967) has suggested that concentration of matter in filamentary structures aligned 
by the magnetic field, exists in the active region before the flare occurrence and becomes visible as a flare if there is a change of heat balance. This may appear when a decompression wave reaches the region, and such a wave can originate through an opening of the magnetic bottle above the active region. Particles can be accelerated to lower energies by a field of random Alfvén waves inside the bottle, and to high energies by the bottle opening. Elliot (1969) has supposed that the energy released in flares comes from energetic protons trapped in sunspot fields. Those particles may acquire their energy over a period of hours or days and the flare phenomenon corresponds to the catastrophic dumping of the particles into the chromosphere.

Hyder (1967) tries to explain the chromospheric flare brightenings, and those of two-ribbon flares in particular, by prominence material lifted out at disparitions brusques and falling down along the field lines into the chromosphere. He supposes that the kinetic energy of fall is then dissipated in the flaring areas. Although the original arguments favouring this model were largely circumstantial, in more recent data on 85 flares Hyder finds $75 \%$ to show rapidly falling material which is intimately associated with bright flare regions. As mentioned in the preceding Chapter, Hyder's ideas also receive support from studies carried out by Bruzek and Janssens and White (1970). Support for this theory also can be found in the studies published by Slonim $(1968,1969)$, who has found many spatial coincidences of fiares and quiescent filaments, both in active regions and away from them. Nakagawa and Hyder have considered the way in which the infalling material modifies the temperature and density structure of the chromosphere, and their results are being used by Athay and Hyder to predict the observed line profiles. More spectroscopic Doppler shift data and polarization measurements are needed to confirm the infall-impact theory.

Other theoretical considerations are aimed at the problem of instabilities and the fast rate of energy dissipation. Sturrock's (1968) model explains the flare by reconnection of magnetic field in a bipolar magnetic-field structure by a tearing-mode resistive instability. The energy stored in the pre-flare closed-field region is derived from photospheric motion. The time-scale of the reconnection process is of the order $10^{2}-10^{3} \mathrm{~s}$ if reconnection proceeds by the Petschek mechanism, with the modification that resistive diffusion is replaced by the more rapid Bohm diffusion. The plasma affected by the reconnection process is believed to be highly turbulent, which would lead to stochastic acceleration of both electrons and protons. Highly turbulent plasma, rendered by current-driven electrostatic instability, is also supposed by Friedman and Hamberger (1969) in Petschek's model of magnetic field annihilation, with plasma flowing through the boundary layer, where its magnetic energy is released. The same authors (Friedman and Hamberger, 1968) have also shown that the region near the neutral point, whose dimensions are governed only by diffusion processes, would be subject to current induced micro-instabilities, which would lead locally to a fast rate of magnetic field diffusion and hence to physically reasonable dimensions of this region. Syrovatskij (1969) supposes that the process of dynamic dissipation of magnetic energy near the zero lines of the magnetic field is the base of the solar flare mechanism. Křivský (1968) has proposed a magnetic model of two-ribbon flares in the form of an interaction of an emerging magnetic channel from the photosphere, entering a field of an already existing interaction of two systems of magnetic fields of a complex active region. According to Schmidt (1969) who summarized the theoretical aspects of the flare phenomenon, the annihilation of magnetic flux in a current sheet combined with a hypothetical hydromagnetic wave mode seems to give the fastest rate of dissipation. The explosive onset of this dissipation may be possible if the energy is stored in the sheared magnetic fields supporting a gradually condensing filament against gravity or in force-free currents induced gradually by the action of convective rolls in the penumbra.

Severnyj's (1969a, $b$; Moreton and Severnyj, 1968; Severnyj and Zvereva, 1969) measurements of the magnetic fields in flare regions have given strong support to Alfvén and Carlqvist's (1967) theory that a solar flare is caused by a sudden interruption of electric currents. The energy of the flare is supposed to be stored as magnetic energy in the current system. If, due to a local constriction at a certain point, the current density exceeds the maximum value which the plasma can carry in a stable way, a local decrease in plasma density is produced and an over-voltage arises in the circuit which will give rise to a rapid release of the stored energy. In the created high impedance region 
ions and electrons can be accelerated to very high energies within a reasonably short time comparable with the flash phase duration of the flare (Carlqvist, 1968).

Acceleration processes in flares have been discussed by De Jager (1968). He has suggested as the most probable case a two-step acceleration, with the Alfven-Carlqvist mechanism as the first, and Fermi acceleration as the second step. Particle acceleration by nonlinear waves (in chromospheric ion solitons) has been suggested by Ginzburg (1967). By means of comparing spectral diagrams of type IV bursts with the particle production, Sakurai (1967c) has concluded that there are two rates of space-time change in sunspot magnetic fields, one of an explosive nature and the other gradual, on which the efficiency of particle acceleration depends. Boischot and Clavelier (1968a) suggest that electrons in the corona can be accelerated by the mechanism described by Tsytovich, if an ascending perturbation coming from the chromosphere attains some critical value of the Mach number.

As the ultimate fiare energy is concerned, Anzer (1968) has proposed that the energy of flares comes from the convective motion in the penumbrae of sunspots. Stenflo (1969a) has shown how the kinetic energy of the rotational motion of a sunspot can be transferred to electromagnetic energy in filamentary currents, thus building up flare energies within reasonable time. Sakurai (1967b) has suggested that a solar flare is the result of a twisting of the magnetic lines of force due to a rotational motion of sunspot groups driven by the upward convective motion near the photospheric surface.

\section{FLARE-LIKE AND FLARE-ASSOCIATED OPTICAL PHENOMENA}

The fine broad spectral features known as moustaches have been discussed by several authors. Bruzek reports a continuing study in which he has measured sizes of over 700 such features, finding angular sizes of 0.5 arc seconds under best seeing conditions and he believes that the true size is, in fact, somewhat smaller. This is not inconsistent with Engvold and Maltby's (1968) study, or the earlier results of Severnyj. Bruzek has also confirmed that there is no conspicuous association between the moustaches and flares. From a study of metallic line emission in moustaches, Koval (1967) has deduced a velocity increase with height. The blue asymmetry, particularly pronounced in lines formed in greater depths, decreases with the moustache development. Asymmetry in the far wings has been studied more in detail by Koval and Severnyj (1970), and Severnyj (1968) explains the asymmetrical line profile as due to incoherent scattering in contracting shells (cf. Subsection 8.1). The continuous emission accompanying moustaches mostly shows rapid growth towards the violet (Koval and Severnyj, 1970).

Flare-like "dashes", first discovered by Prokof jeva more than $10 \mathrm{yr}$ ago, have been studied by Krat (1968), who considers them for a separate class of flare-like objects closely connected with spicules. Kiepenheuer's (1968c) suspicion that surges originate in the photosphere or very close to it, in close connection with the granulation, might indicate, that there is some connection among all the three phenomena, spicules, dashes, and surges.

A review paper summarizing the flare-associated optical phenomena was written by Bruzek (1969). A number of expanding coronal phenomena associated with flares have been found by Bruzek in recent Sacramento Peak coronal movies taken in $\lambda 5303 \AA$. In particular: a slow and decelerated expansion of concentric coronal arches $(v<10 \mathrm{~km} / \mathrm{s})$, and a fast and accelerated expansion of coronal arches ( $\geqslant 150 \mathrm{~km} / \mathrm{s}$ ) which terminates by the explosion of the arch. The nature of flare sprays and their association with other solar phenomena has been discussed by Elske Smith (1968). Ogir (1969) has shown that sprays from two flare knots situated in regions of opposite polarity are directed towards each other and may lead to the formation of a loop. Smith, however, has concluded that sprays by themselves do not instigate other interesting phenomena, which perhaps is a disappointing conclusion when one considers how spectacular some of these events are. They may be related to surges, but are almost an order of magnitude more energetic. This recalls McKenna's (1967) remark, pointing out that the various active phenomena associated with an active region appear to require very different energies, so that these events may be considered as monitors of the magnitudes of the accelerating disturbances which initiate them. 
Dodson and Hedeman (1968e) and Zirin and Russo Lackner (1969) have described a large expanding feature which moved from the site of the proton flare of 1966 August 28, with a velocity of about $700 \mathrm{~km} / \mathrm{s}$. A similar phenomenon also was observed in association with the flare of 1967 May 23 (Dodson and Hedeman, 1969). The velocity was $\sim 1000 \mathrm{~km} / \mathrm{s}$. These flare waves seem to be the visible manifestation of disturbances which cause the disparitions brusques. Models for such wave disturbances following flares have been presented by Uchida (1968) and Meyer (1968). Labrum and Smerd (1968) have discussed two events, in each of which it appears that shock waves arising from a flare produced distant prominence activity associated with type IV continuum. A similar event was also discussed by Wild et al. (1968) and Wild (1969). On the other hand, Křivský (1969) has interpreted these results as due to a moving radio type IV condensation, having its origin in the flare itself.

\section{PROMINENCES}

(by J. T. Jefferies, University of Hawaii)

Observations and analysis of spectral line intensities and shapes in prominences have been reported by Geichenko, Morozhenko, Sergejeva, Smotrelkina and Zeldina in several papers published in the Volume Voprosy Astrofysiki (Porfirov, 1967). Characteristic of much of the newer work is the recognition that a filamentary model may be needed to explain the spectral data and that a satisfactory model may need also to include temperature and density variations. An exhaustive study of 8000 emission line widths of four quiescent prominences whose spectra were obtained at the 1966 total eclipse is reported by Hirayama who concluded that kinetic temperature is around $6000 \mathrm{~K}$ inside and $12000 \mathrm{~K}$ near the edge of the prominence; the turbulent velocity varies from $8 \mathrm{~km} / \mathrm{s}$ inside, to $20 \mathrm{~km} / \mathrm{s}$ on the edge, the HeI emission originates in the low temperature regions, and the electron density is about $4 \times 10^{10} \mathrm{~cm}^{-3}$. Further work is aimed at obtaining a two-dimensional model of the filament. These results, where comparable, appear to be characteristic of the results obtained by Stellmacher (1969).

Leroy $(1967,1969)$ has obtained monochromatic filter (10 $\AA$ bandpass) images of prominence in the light of $\mathrm{HeI}, \mathrm{HeII}, \mathrm{H} \alpha$ and Fex, exposed so as to allow particular study of the faint peripheral prominence regions. The similarity of the $\mathrm{HeI}$ and HeII images gives support to the idea that $\lambda 4686$ (He II) is excited by coronal radiation as Falciani and Rigutti (1967) have tentatively suggested. Leroy has been able to photograph extremely faint emissions extending far above the limb using this coronagraph technique. Future studies of the motion and disappearance of such faint wisps, which he plans, should be very interesting. Similar work is in progress in Hawaii, where McCabe and Fisher have completed a detailed study of the motion of the eruptive prominence of 1969 March 1, where $\mathrm{H} \alpha$ emission was seen extending beyond a solar radius. Similar work is also reported to be in progress at Kandilli by Soytürk using isophotal contours of $\mathbf{H} \alpha$ photographs of a surge to study morphological changes.

The microstructure of prominences is still not satisfactorily understood either in terms of the range of temperatures and densities present or the degree to which prominences exhibit subtelescopic filamentary structure. Much more refined studies of the excitation of the prominence gas are needed, as are spectroscopic observations at high spatial resolution at coronagraph stations. Measurements of the wing shapes of Balmer and other lines could give valuable information as to the mechanism of excitation. Such studies should also consider the influence of motion on the visibility of $\mathrm{H} \alpha$ prominence filtergrams, as Rompolt (1967) has done in a preliminary way.

Öhman et al. (1968) have drawn attention to the frequent occurrence in spectra of quiescent prominences of slanted spectral lines, and of "lines" with a circular appearance, and with other peculiar forms. Similar phenomena are well known in flares, moustaches and spicules. The standard interpretation in terms of a rotating structure has been broadened by Öhman to a self-twisting toroid (like a smoke ring). In this connection it is interesting that observations of eruptive material in prominences and surges are frequently strongly suggestive of an untwisting motion (Valníček, 1968; Kleczek, 1968; Dizer, 1968; Valníček et al., 1969). Detailed spectroscopic studies of such twisting and untwisting give promise of elucidating the general questions of factors underlying prominence 
stability and instability. A model for quiescent filaments with helical structure has recently been completed by Anzer and Tandberg-Hanssen (1969). The structure arises from the combination of a pair of fields, one having a photospheric source, the other deriving from currents along the cylindrical filaments. This model is similar in nature to one suggested earlier by loshpa (1968).

Suggestive in this context, too, is the tentative association by Liszka (1968) between the amplitude of small scale prominence motions and the degree of activity in the neighboring photosphere. A more refined study which accounts roughly for his observed relation between field strength and random velocity amplitude is given by Malville (1968) who suggests that these persistent motions derive their source in oscillations of field lines which are, in turn, driven by motions in the denser photospheric layers. Large amplitude horizontal oscillations, giving rise to the "winking" filaments, are studied by Kleczek and Kuperus (1969). They attribute the dissipation of the oscillations to the generation of acoustic waves in the surrounding coronal plasma.

Bruzek $(1967,1969)$ has studied in detail the $\mathrm{H} \alpha$ arch filament systems closely associated with bipolar spot groups. Material is found to be moving at high speed $(50 \mathrm{~km} / \mathrm{s})$ down both sides of the arches and upward (at $10 \mathrm{~km} / \mathrm{s}$ ) at the tops. Bruzek suggests that these systems are produced by expansion of the spot field with a consequent elevation of material into the chromosphere where it slides down field lines under gravity. They are not related to flare occurrence.

Some very interesting measurements of the line-of-sight component of the magnetic field in prominences above the limb have been reported by a number of investigators: Malville (1968), Ioshpa (1968), Rust (1967), Malville and Tandberg-Hanssen (1969), Harvey and Tandberg-Hanssen (1968), and Lee et al. (1969). The results seem generally consistent, giving fields characteristically of about $6 \mathrm{G}$ for quiescent and about $30 \mathrm{G}$ for active prominences with wide variations around these values - fields up to $200 \mathrm{G}$ have been reported. It is found that lines of various elements all give essentially consistent values. Smolkov and Bashkirev (1969) have studied line profile parameters in spectra of quiescent prominences in order to derive the magnetic field configuration in the prominence region, and Smolkov (1969) also followed the time evolution of the longitudinal component of the prominence magnetic field and its relation to the redistribution of brightness. Von Klüber (1967) has measured the field variation across filaments confirming their preferred occurrence along neutral lines. According to Harvey (1969), the typical prominence field is characterized as having a non-zero component normal to its axis and a monodirectional field along the entire length.

The formation of solar prominences from coronal gas has been considered by Raju (1968) for a uniform magnetic field distribution. He finds that, although the instability leads to a strong temperature decrease, with a time scale of order $10^{6} \mathrm{~s}$, it would result in prominence densities not significantly greater than those in the surrounding corona. Kuperus and Tandberg-Hanssen (1967) have discussed the condensation problem also, but have adopted a field geometry more in keeping with observation, in which the prominence is formed along a neutral sheet. They showed that this is a thermally unstable region which, through cooling of the matter, results in the field lines being drawn together with consequent compression and radiative cooling in a characteristic time of $10^{5} \mathrm{~s}$. In order for this situation to be preserved against heat conduction from the corona, however, these authors suggest that the magnetic field in the region of the neutral plane must become unstable (a tearing mode instability) and break up into tubular filaments of characteristic size $\sim 300 \mathrm{~km}$. Of very great interest to such theoretical studies would be observational studies aimed at following the formative period of prominence material in condensing from the hot coronal gas.

Other theoretical work of interest in prominence formation has been carried out by Anzer (1969) who has obtained necessary and sufficient conditions on the magnetic field gradients for the Kippenhahn-Schlüter mechanism to apply. His stability analysis leads to the early conclusion of Kippenhahn and Schlüter that the horizontal component of field strength increases with height, which has been confirmed by Rust (1967) for many prominences, and that the vertical component of the field at the filament boundary should decrease with height. The nature of prominences is also being further investigated by Sturrock, with the aim of determining the magnetic field structures of prominences and the role of thermal instabilities in these objects.

The reader's attention also is drawn to the proceedings of the 9th Nobel Symposium (Öhman, 
1968), which contains a valuable collection of articles dealing with the measurement and analysis of motions in prominences, sprays and surges.

\section{CORONAL CONDENSATIONS}

(by G. Newkirk, Boulder*)

The past three years have seen extensive work on the study of coronal condensations, especially in the UV and X-ray regions (Goldberg, 1967; Neupert, 1969). Not only have these new observations permitted the construction of detailed models of the electron density and temperature structure, but they have also yielded information on the temporal evolution of a "typical" such region (Neupert, 1969). These details generally confirm the ideas derived from earlier, optical observations of the corona.

According to the UV and X-ray observations, the typical condensation exists at a temperature of from 1.8 to $2.4 \times 10^{6} \mathrm{~K}$, has an electron density of approximately 3 or $4 \times 10^{9} \mathrm{~cm}^{-3}$, a volume of about $10^{29} \mathrm{~cm}^{3}$, and an emission measure, $N_{\mathrm{e}}^{2} V \sim 10^{48}$. Within such a region, temperatures up to $4 \times 10^{6} \mathrm{~K}$ and densities up to $7 \times 10^{9}$ may occur. Such a region appears to correspond well to the permanent and sporadic condensations visible in the green and yellow coronal lines and described in the earlier literature.

The evolution of a typical such condensation shows a rapid increase in the electron density during the first two weeks of development of the underlying active region (Neupert, 1967). At about the time when the region reaches maximum activity the temperature also reaches its maximum of approximately $2.5 \times 10^{6} \mathrm{~K}$. That the temperature declines more rapidly than the emission measure is demonstrated by the fact that the lines of cooler coronal ions (FexIV and FexII) reach a maximum a week or two after the maximum in such ions as FexvI.

The systematic investigation of a wide variety of lines originating from active regions (Noyes et al., 1969) has allowed not only the determination of the average conditions in the corona and chromosphere-corona transition region, but also a measurement of the conductive flux in such regions. These observations show a typical active region with a temperature of approximately $2.5 \times 10^{6} \mathrm{~K}$ and enhancement of about 5 times in the pressure in the chromosphere-corona transition region, and electron density enhancement of approximately 4 times, and a conductive flux from the corona back into the chromosphere increased by about 5 times.

There appears to be some indication of a disagreement in the conditions in coronal condensations inferred from the spacecraft observations and inferred from optical emission line observations in the direction of the short wavelength observations requiring somewhat higher electron densities and a rather higher prevalence of temperatures of $3-4 \times 10^{6} \mathrm{~K}$ than would be suggested by the optical observations. In the absence of the detailed study of the consistency of the optical, radio, UV, and $\mathrm{X}$-ray observations of particular regions in the corona, it is unknown whether this apparent disagreement is real or not. It may well reflect the fact that the short wavelength observations preferentially see the densest and hottest regions and that the optical observations seldom give reliable information concerning the lowest portions of the corona.

Another disagreement which appears to have arisen is that of the maximum temperature which is present in coronal condensations. Optical observations would most certainly place this maximum at $4-5 \times 10^{6} \mathrm{~K}$. However, UV and X-ray measurements (Hudson et al., 1969; Neupert, 1967; Neupert, 1969) which show the spectral lines up to FexXV require the presence of material with temperatures of up to $3 \times 10^{7} \mathrm{~K}$ even in the absence of reported solar flares. At the present time it is not known whether this radiation originates in the corona or whether it represents the impact of non-thermalized particles of energies of $10-15 \mathrm{keV}$ into the lower solar atmosphere. A strong case has been made that, for solar flares at least (Acton, 1968), the observations are best described by a corona which has a maximum temperature of $4-5 \times 10^{6} \mathrm{~K}$ and a substantial nonthermal component.

- A more extensive draft available on request. 
In the future we can expect spacecraft observations with good angular resolution to play an increasing role in the investigation of coronal condensations. Because the regions can be followed completely across the solar disk, the evolution and response of the condensation to such cataclysmic effects such as flares can be documented in a manner hitherto impossible. Since these measurements also allow conditions in the crucial chromosphere-corona transition region to be examined, we can expect detailed knowledge of the change in energy transport in these regions as well. Hopefully, satellite and ground-based observations of particular regions can be combined to further elucidate the details of the temperature density structure in such regions.

The problem of explaining the high temperature of the solar corona has received considerable attention in the past several years. Most of the theories (for a review see Kopp, 1968; Kuperus, 1969) have been concerned with the details of the generation of mechanical energy in the lower solar atmosphere and the deposition of this energy by shock waves in the chromosphere and corona. Recently the role of ion acoustic waves, which dissipate their energy by Landau damping in the lower corona, has been pointed out by d'Angelo (1969). Perhaps, the most important aspect of these theoretical investigations has been their demonstration of the fact that the magnetic field in the lower atmosphere can be expected to play a very large role in determining the rate of energy deposition in the corona as well as the structure of the chromosphere-corona transition region (Kopp and Kuperus, 1968). As mentioned in the section on coronal condensations, there now appears to be substantial evidence (Noyes et al., 1969) that the energy deposition into the corona is, in fact, increased substantially above active regions.

Temperatures in the outer corona above active regions have been inferred from the duration of type III radio bursts for several years. The advent of space-borne radio receivers operating at frequencies down to $0.4 \mathrm{MHz}$ has extended these observations out to what appears to be $30 R_{\odot}$ (Alexander et al., 1969; Hartz, 1969; Slysh, 1967). The usual interpretation of these observations is that the type III burst radiates at the local plasma frequency, that the duration of the burst is determined by the local collision frequency and that the local temperature can thus be obtained. The relation of temperature to distance from the sun is then derived by assuming a density model. If the density over the active region is taken to be some constant times the ordinary coronal models or even includes a simple evaluation of the role of the magnetic pressure in influencing the density structure, the temperature is found to fall off much more rapidly than would occur in the conduction model of the corona. Such observations would then suggest that the streamers over active regions are dense and cold compared to the rest of the corona. In fact, they would appear to be in subsonic expansion (Newkirk, 1967b). An alternate hypothesis (Alexander et al., 1969) is that the temperature in the active region streamer is the same as in the coronal background, which is taken as that of the solar wind heated by conduction from below. The question of subsonic streamers above active regions is then avoided. Such a streamer would have a maximum density enhancement of about 5 times the background at about $30 R_{\odot}$. It should be noted that this is approximately the density and temperature behaviour which is predicted by the theoretical streamer model of Pneuman and Kopp (1970). Recent evidence (Warwick, 1967), suggests that local heating by strings of type III bursts far out in the corona is required to explain the appearance of type $\mathrm{V}$ radio bursts.

An examination of the rotation of coronal condensations during the period 1964 to 1967 (Hansen et al., 1969) demonstrates that the rotation rate varies from year to year and in any given year may be different between the two hemispheres. At lower latitudes, the rate appears to agree with that determined from filaments and sunspots while at high latitudes the rate appears to be similar to that of filaments, which is over $2^{\circ}$ per day faster than that displayed by polar faculae. These data show a dependence of angular velocity with height which appears to depend upon both latitude and the phase of the solar cycle. Both increasing and decreasing angular velocity with height appear. The details of this phenomenon are poorly understood. These is some indication that the high latitude coronal features are being influenced by the more rapidly moving magnetic centers at lower latitude.

In spite of the fact that the determination of magnetic fields in the corona is extremely difficult, the question of such fields and their influence on the solar corona has been one of tremendous activity in the past several years. Although, in theory, capable of yielding information on these fields, the 
measurements of coronal emission line polarization (Eddy and Malville, 1968; Hyder et al., 1968) have proved difficult to interpret in terms of the magnetic field in any detailed manner. Faraday rotation of artificial and natural radio sources (Levy et al., 1969) is also a potentially useful tool although no quantitative measurements of the coronal field have yet been determined. The Razin effect in solar radio emission (Boischot and Clavelier, 1968b; Ramaty and Lingenfelter, 1968; Bohlin and Simon, 1969) appears to have given us the most unambiguous determination of the field at a value of 0.3 to $0.5 \mathrm{G}$ at $2 R_{\odot}$ above an active region. All these researches refer to the observation of a single type IV burst and similar observation of other events is urgently required. A similar value has been inferred from the weak polarization of type III bursts (Kai, 1970). At the end, one has to emphasize that the observation of radio bursts at $80 \mathrm{MHz}$ per second with high angular resolution with the Australian radioheliograph has opened an entirely new vista in the study of magnetic fields and high energy particles in the corona (Wild, 1968a, 1969a, $b$; Kai, 1969, 1970).

\section{RADIO ASPECTS OP SOLAR ACTIVITY}

(by A. D. Fokker, Utrecht)

(See also the section Solar Radio Emission of the Commission 40 report).

The past three years are distinguished by a further improvement of observational instrumentation and by some coordinated efforts to collect the largest possible material for some selected solar events. Progress has been made in the treatment of gyro-synchrotron emission and a new element in theoretical discussion was the attention paid to the possible influence of the Razin-effect on the shape of burst spectra. Certain notions about the radiation mechanism of type II and type III bursts have become rather generally accepted, but the detailed treatments of processes cannot yet be summarized in a textbook kind of presentation which could be understood by everybody. The occurrence of type I storms is so common as to make people forget that these, in particular, are still ill-explained. Two review papers on the theory of radio bursts and on the generation of radio waves in astrophysical plasmas, respectively, have been written by Takakura (1967) and by Smerd (1968).

\subsection{Progress in equipment}

The main instrumental achievement in interferometry has been the completion of the $80 \mathrm{MHz}$ Culgoora radio heliograph (Wild, 1967), which consists of 96 dishes each of $13 \mathrm{~m}$ diameter and equally spaced around a circle of $3 \mathrm{~km}$ diameter. Sidelobes are suppressed by connecting the aerials with each other via different phase lags according to a fast computer-controlled sequence. The pencil beam is made to scan the Sun two-dimensionally along a grid of $60 \times 48$ points and a two-dimensional picture of the Sun with a resolving power of $3.5^{\prime}$ is displayed twice a second on a cathode ray tube.

The 16-element array of the $169 \mathrm{MHz}$ Nancay interferometer has been provided with a system of coaxial cables which permits simultaneous reception of solar radiation in 31 equidistant fan beams. With this multibeam system instantaneous one-dimensional pictures are obtained (Vinokur, 1968). At Toyokawa, the $(32+2)$-element compound interferometer for $3750 \mathrm{MHz}$ with a half power beam width of $23^{\prime \prime}$, completed in 1967 , has been provided with rotary phase shifters which permit the Sun to be scanned once every $10 \mathrm{~s}$ with a 1:1 beam (Tanaka et al., 1969b). Two-dimensional mapping of the Sun at $\lambda 3 \mathrm{~cm}$ with a $32 \times 16 \mathrm{EW} / \mathrm{NS}$ array takes $20 \mathrm{~min}$ and the pencil beam measures 1'.6 $\times\left(1{ }^{\prime} 6-3.2\right)$. At Algonquin Observatory, an array of 32 reflectors for operation at $2800 \mathrm{MHz}$ has been completed and yields a half power width of 1.5 (Covington et al., 1967). Four additional reflectors will be added to form a compound interferometer with a beam of 0.25 width.

High-resolution $\left(35^{\prime \prime}\right)$ fan-beam scans at $\lambda 21 \mathrm{~cm}$ are obtained at Fleurs, Australia with a 32-element array. It is intended to develop there a fast-scanning device which permits a continuous display 
to be obtained of the Sun's radio profile. A new $(8+3)$-element compound interferometer operating at $160 \mathrm{MHz}$ with a resolving power of 1.8 in the $\mathrm{E}-\mathrm{W}$ direction was completed by Tokyo Astronomical Observatory in 1969. The fan beam is made to sweep the Sun once in $\mathbf{0 . 1} \mathrm{s}$. At Stanford University, a new radio telescope is under construction, with a resolution of 20 seconds of arc and with provision for polarization measurement.

A novel type of radiospectrograph was put into operation by the Utrecht Observatory. The octave from 160 to $320 \mathrm{MHz}$ is covered by 60 equidistant single-frequency channels. The radiospectrograph displays the instantaneous intensities as referred to a level which is the intensity averaged over the past three or ten seconds, so that relatively small fluctuations are reproduced with considerable contrast (De Groot and Van Nieuwkoop, 1968). At Fort Davis, a new sweep-frequency receiving equipment, covering the band $50-2000 \mathrm{MHz}$ is being put into operation with an $85-\mathrm{ft}$ paraboloid antenna. A 64-channel spectrometer with a time resolution of $0.001 \mathrm{~s}$ for the range $400-1420 \mathrm{MHz}$ is being developed by the radio observatory of Kiel University. A swept receiver from about 310 to $340 \mathrm{MHz}$ has been in use since 1967 at Harestua, and from 50 to $200 \mathrm{MHz}$ since 1968 at Ondřejov and Irkutsk. For low-frequency spectrographic work aboard satellites cf. Subsection 2.3.

At Harestua, a receiver at $159 \mathrm{MHz}$, working since summer 1968 , has been specially constructed for the recording of extremely short-lasting solar bursts. At the Heinrich Hertz Institute in Berlin, improved polarization measurements are now available at 23,30, 40 and $115 \mathrm{MHz}$, and in 1971 polarization measurements also will be carried out at 793, 1470 and $9500 \mathrm{MHz}$.

\subsection{Observations of radio events}

More detailed information on radio events has become available thanks to the diversification of world-wide observations and to the progress of instrumental sophistication.

High resolution observational results on microwave bursts obtained at Toyokawa were reported by Énomé et al. (1969). A change in polarization was observed by Tanaka and Énomé during a fairly strong simple-structured outburst and several cases of multi-source bursts have been recorded both by drift-scanning and quick-scanning. The source size of the impulsive burst was found of the order of 0.5.

Observational results with the Culgoora radio heliograph were reported for several events. The heliograph has revealed interactions between distant centers around the Sun (Wild et al., 1968) and established a new kind of phenomenon in which two distant sources appear within a few seconds or even within one second of one another (Wild, 1968). The magnetic structure of a type IV event could be inferred from total radiation and polarization images (Wild, 1969b). An expanding arch structure of a great solar radio outburst has been described by Kai (1970). Several reports on other observations made with this equipment are contained in Vol, 1, nos. 4 and 5, of the Proceedings of the Astronomical Society of Australia.

Detailed observations of spectrum and position of a weak moving type IV event on 1966 September 14 were reported by Boischot and Clavelier (1968b). This event has received a lot of discussion, by Boischot and Daigne (1968), Lacombe and Mangeney (1969) and Ramaty and Lingenfelter (1968). The shape of its spectrum seems to be strongly influenced by the Razin effect. A strong stimulus for making multifixed frequency observations of solar radio events is provided by the widespread interest for solar particle events (Švestka and Simon, 1969). Castelli et al. (1967) noted that U-shaped spectra of type IV bursts (with minimum flux around $2000 \mathrm{MHz}$ ) are indicative for production of high-energy particles. Extensive observational material of the Heinrich Hertz Institute (Berlin) on several big radio events was presented and discussed by Böhme and Krüger (1968).

Burst observations in the very low frequency range, made with satellites, have been reported and discussed by Slysh (1967), Hartz (1969) and Alexander et al. (1969). On the other hand, solar radio observations have been pushed through to higher frequencies so as to encompass the $\mathrm{mm}$ wavelength range. Some bursts at $\lambda 3.3 \mathrm{~mm}$ were reported by Shimabukuro (1968), who found good correlation with solar flares. These bursts reached relatively low intensities, in contrast with an outstanding solar radio burst at $\lambda 4.2 \mathrm{~mm}$ reported by Croom and Powell (1969), which reached 
a peak intensity of $47 \times 10^{-19} \mathrm{Wm}^{-2} \mathrm{~Hz}^{-1}$. This outburst was associated with a proton flare. Infrared techniques were employed by Clark and Park (1968) to map the sun at $\lambda 1.2 \mathrm{~mm}$. In their $3^{\prime}$ beam they observed transient enhancements of brightness temperature of the order of $100 \mathrm{~K}$, which apparently were associated with certain filaments.

\subsection{Application of the theory of gyro-synchrotron emission to microwaves and type IV bursts}

Computations of the spectrum and time variation of microwave and type IV bursts have been carried through in more and more detail. Gopasjuk et al. (1967) discussed the role of compression and expansion of the region that generates synchrotron radiation, in order to explain microwave bursts of the explosive type. Takakura et al. (1968) dealt with the time variation of the spectrum of gyro-synchrotron emission, assuming a power-law distribution of electron energies. In a paper by Holt and Ramaty (1969) detailed theories of gyro-synchrotron emission, the Razin effect, gyroresonance absorption and gyro-synchrotron reabsorption were applied, and for a given set of parameters the overall spectrum of the type IV event associated with the proton flare of 1966 July 7 , could be reproduced.

Whereas the above authors based their computations on fixed values of the magnetic field and the electron density in the source region, Yip (1967) interpreted the properties of type IV bursts on centimetre and decimetre wavelengths on the basis of a model for the electron density distribution and the magnetic field configuration, using theories of Doppler-shifted cyclotron radiation, its excitation in a stream-plasma system and of gyro-resonance absorption. Kai (1968a) discussed evolutional features of microwave type IV bursts and made estimates of the strength of the magnetic field and the energy distribution function of electrons in the source regions where subsequent peaks in one and the same event originate. Ramaty and Lingenfelter (1968) interpreted the spectrum of the type IV burst of 1966 September 14, which originated high in the corona, in terms of gyrosynchrotron emission with due allowance for the Razin effect. A value of the magnetic field and the range of electron energies were derived.

\section{SOLAR EUV RADIATION, X-RAYS AND GAMMA RAYS}

(by E. v. P. Smith, University of Maryland)

This subject was recently reviewed in an excellent article by Neupert (1969). Since space is limited, we will largely avoid recapitulating material covered in that review paper. A vast amount of data has accumulated from OSO 3 through OSO 6 spacecraft, and in fact is continuing to be accumulated especially by OSO 6 . This material is supplemented by data obtained from rocket flights, which usually have the advantage over satellite data in having far better spatial resolution. Among the outstanding results one should mention the localized nature of the flare X-ray emission and the large number of lines in the soft $X$-ray region of the spectrum which become prominent at times of flares.

The group at American Science and Engineering (Vaiana et al., 1968) obtained high resolution $\mathrm{X}$-ray photographs of the Sun at the time of the $1 \mathrm{~N}$ flare of 1968 June 8, showing considerable fine structure in both the flare and in active regions. A very close correspondence has been found between $\mathrm{H} \alpha$ and $\mathrm{X}$-ray features, but the X-ray regions extend well above the $\mathrm{H} \alpha$ counterparts at the limb. The brightest part of the X-ray flare was located at the position of a filament that had disappeared minutes earlier.

Many soft X-ray flare lines are observed in the region from 1 to $20 \AA$ (Neupert et al., 1967, 1969; Meekins et al., 1968; Evans and Pounds, 1968; Evans et al., 1969; Jones et al., 1968). A large number of these have been identified by Gabriel and Jordan (1969) with the resonance, intercombination and forbidden lines of helium-like ions such as OVII, NeIx, MgXI, and SixIII. Neupert and Swartz (1969) have added to the identification other transitions of this type along the helium isoelectric sequence up to NixXVII. Gabriel and Jordan showed how the relative intensities of these triplet lines can be used to calculate electron densities of the emitting region, while the temperatures obtain 
from the presence of a particular stage of ionization. They find that temperature and density increase together, and the densest regions tend to be the most localized.

The dominant spectral line below $3 \AA$ is that near $1.9 \AA$. This has been observed both by Goddard and NRL groups at the times of flares but they disagree on its identification. Neupert (1969) argues that it is Fexxv while Meekins et al. (1968) prefer to identify the line with inner-shell transitions. The fact that this line reaches maximum intensity earlier than lower ionization lines has led Neupert to suggest that a very hot plasma exists or is ejected into the corona at the start of a flare and the lower ionization lines arise as the plasma cools and recombination occurs. Since a large range in ionization stages is observed simultaneously, it is evident that a wide range of temperatures must exist at a given time. Relative intensities of continuum to line energy show that the continuum emission is about a factor 10 greater than the integrated line emission during flares (Meekins et al., 1968; Neupert et al., 1969). This continuum radiation is "hardest" near the maximum of the X-ray flare.

The University of Leicester group under K. Pounds (Evans et al., 1969) has observed X-ray flares in the 1 to $18 \AA$ range on OSO 4 with a time resolution of $16 \mathrm{~s}$. The bursts range from being very impulsive with 1 to 2 min duration to long enduring events of several hours. Many events have a precursor in the form of a weak gradual increase in X-radiation, corresponding to a temperature about $8 \times 10^{6} \mathrm{~K}$ some minutes before the flare event. Kane and Winckler (1969) also find that X-ray bursts have two components, an impulsive and a more slowly varying one. A catalog of $2-12 \AA$ solar X-ray flux is being produced and has been completed for the period from July 1966 to September 1968 by Drake et al. (1969), based on the measurements with the University of Iowa equipment on Explorers 33 and 35.

In contrast to the thermal or quasi-thermal character of soft X-rays, the higher energy or hard X-ray bursts are nonthermal (Cline et al., 1968; Arnoldy et al., 1968a; Hudson et al., 1969a). Frost reports on the OSO 5 observations in the 14 to $250 \mathrm{keV}$ energy range of the 1969 March 1 $\mathrm{X}$-ray burst. Remarkable fine structure in the intensity-time profile was revealed by all 9 channels within this energy range, but especially at the lower energies. The intensity fluctuations closely followed the microwave burst structure. On the other hand in the flare of 1968 July 8, Van Allen and Wende (1969) have found only a general association of soft X-ray and centimeter radio flux, with lack of detailed similarity. Frost (1969) also has found that both X-rays and radio bursts were quasi periodic with a period of about $35 \mathrm{~s}$. Similar, 10-s periodic pulsations, with X-ray spectrum hardening during the peaks, which also correlated well with the microwave data, have been observed by Parks and Winckler (1969) at the flare of 1968 August 8.

An Atlas of 10-50 keV Solar Flare X-rays observed by the OGO satellites from September 1964 to the end of 1967 has been published by Arnoldy et al. (1968b) and Kane and Winckler (1969b). Another catalog of X-ray bursts with energies in excess of $7.7 \mathrm{keV}$ is being prepared by Petersen and his colleagues, based on OSO 3 observations. They find that nearly all $\mathrm{H} \alpha$ flares and subflares produce X-rays above $7.7 \mathrm{keV}$, but a significant number of bursts do not seem to have corresponding $\mathrm{H} \alpha$ events. Generally, while the time development of soft X-rays closely follows the $\mathrm{H} \alpha$ flare variations, the duration of harder X-ray bursts becomes progressively shorter and peaks earlier, before the $\mathrm{H} \alpha$ maximum, as the X-ray energy increases (see, e.g. Svestka and Simon, 1969). Good correlation is found between these bursts and first maxima of microwave radio bursts, while $X$-ray and radio bursts late in the flare development do not correlate, and the correlation with type III bursts is much lower as if not all X-ray bursts affect the upper corona (Arnoldy et al., 1967; Hudson et al., $1969 a$ ). As to the height of the X-ray emitting region, both Beigman et al. (1969) in an X-ray flare and Broadfoot (1967) for X-ray emission from three coronal condensations find $20-25 \times 10^{3} \mathrm{~km}$ above the photosphere.

Active regions were observed over a wide range of wavelengths $(\lambda \lambda 280-1360)$ by the OSO 4 Harvard spectrometer-spectroheliometer (Noyes et al., 1968; Hearn et al., 1969). Because of the wide wavelength coverage it was possible to obtain data pertaining to heights in the solar atmosphere from the low chromosphere to the corona. The enhancement of emission lines in non-flaring regions increases with the ionization temperature. Analysis of spectroheliograms in the $\lambda 537$ and 
$\lambda 584$ lines of neutral helium comparing quiet and active regions shows that increased intensities can be explained purely in terms of increased electron density up to a factor 2.5 . During flares many lines show an intensity increase of a factor 5 or more at maximum. As a rule, pre-flare intensities prevail within $20 \mathrm{~min}$, for the flare duration is shorter in the UV lines than in $\mathrm{H} \alpha$. Hall and Hinteregger (1969) have used an extreme ultraviolet spectrometer aboard the OSO 3 satellite to measure flare-associated enhancements in the wavelength range from 260 to $1300 \AA$ over a six months period. They have found very complex phenomena, the elaboration of which is still in progress. Kreplin and McClinton (Friedman, 1969) and Monsignori Fossi et al. (1969) have observed short-lived enhancements of the UV continuum close to, but outside of the $L \alpha$ line, in the flash phases of the flares of 1966 August 28 and March 20, respectively (also see subsection 8.4). Lemaire (1969) has obtained profiles of the ultraviolet MgII lines with good spatial and spectral resolution, including active and spot regions, while Fredga (1969) obtained spectroheliograms in these lines. Allen (1969) has discussed systematic photometry of XUV solar images. A review paper on twenty years of extreme ultraviolet solar research has been written by Tousey (1967).

The importance of polarization measurements of X-ray bursts below $8 \AA$ in the initial phase of a flare, which can distinguish bremsstrahlung from synchrotron and Compton radiation, has been emphasized by Korchak (1967) and Elwert (1968). A model of hard X-ray and microwave bursts has been proposed by Holt and Ramaty (1969), mostly derived from observations of the 1966 July 7 event (Holt and Cline, 1968). Both kind of bursts are shown to be consistent with a single source of non-thermal electrons.

\section{HIGH ENERGY PARTICles}

The limited extent of this Report does not allow us to touch all the interesting observations carried out in space and in the Earth environment and all the conclusions drawn from them. From the point of view of solar physics, however, three kinds of observation are of particular interest: the discovery of permanent fluxes of $\sim 1 \mathrm{MeV}$ protons from particular active regions on the Sun, records of electrons in space, up to relativistic energies, and an evidence of particle flux arriving to the Earth from the invisible solar hemisphere.

Enhanced proton fluxes in the energy range 0.6 to $13 \mathrm{MeV}$ have been observed to come from the Sun continuously over heliocentric longitude ranges as great as $180^{\circ}$ in association with specific active regions (Fan et al., 1968). An explanation of these extremely wide cones have been suggested by Jokipii and Parker (1969) in term of random fluctuations of magnetic lines of force which lead to rapid diffusion of energetic particles across the average magnetic field. The enhanced fluxes exhibit definitive onsets, when the active region is $60^{\circ}$ to $70^{\circ}$ East of the central meridian, and cutoffs, when the center reaches $100^{\circ}$ to $130^{\circ}$ West (Pioneer 6 and 7 measurements). Superposed on this permanent flux level are occasional large and discrete flare-produced intensity increases from these centers of activity. McCracken (1969), in his very instructive review paper on solar particle events, has expressed an opinion that the generation of energetic particles is a normal feature of all solar flares; nevertheless, as Svestka and Simon (1969) have shown, some necessary conditions have to be fulfilled in the active region to make it capable of producing significant particle events, the permanent particle emission probably being one of them.

Measurements of protons in the energy range of the order of $1 \mathrm{MeV}$ on Soviet space probes Zond 3 and Venus 2, 3, 4 from 1965 to 1967, as well as preliminary results from Venus 5 and 6 have been summarized and presented by Vernov et al. (1969). An extensive study of long-lived streams of lowenergy solar electrons and protons has been made by Anderson (1969). He has suggested that the energetic storm particles, delayed flare events and fluxes associated with active regions, are all probably the same basic phenomenon viewed in somewhat different ways depending on the age of the region and its location on the solar disk.

Electrons with kinetic energy $>40 \mathrm{keV}$ in space were studied by Lin and Anderson (1967). They have found that such electrons follow many solar flares, promptly or delayed, and distinguish two types of the prompt events: those coming from flares beneath large radio noise regions, when electrons are emitted into fairly wide cones of propagation, and all the other events, when the cone of 
propagation is very narrow, with only about $30^{\circ}$ opening angle. Solar flare electron events do not show filamentary structure even at times when protons from the same flare do. And these electrons also are only mildly anisotropic, while low-energy protons coming later from the same flare usually show a sharp anisotropy (Rao et al., 1969).

Arnoldy et al. (1968a) have found a strong correlation between interplanetary solar flare electrons and X-ray bursts, while this correlation is weaker for solar proton events. They also have inferred a strong propagation asymmetry for flare electrons along the spiral interplanetary magnetic field, but the most unusual propagation feature is that the electron flux in delayed particle events is centered several hours before the corresponding proton flux, as has been discovered by Anderson (1969). Jokipii (1969) has shown that at least a part of this separation may be due to difference in drift velocities of protons and electrons in space. Grigorev et al. (1969) and Lin (1969) have reviewed the most up-to-date results on the electron events. They incline to the conclusion that the acceleration and emission of $\sim 40 \mathrm{keV}$ electrons is a phenomenon distinct from the production of solar protons and relativistic electrons.

Relativistic electrons with energy $>3 \mathrm{MeV}$ were detected in space aboard the IMP satellites by Cline and McDonald (1968). The first clear event was associated with the proton flare of 1966 July 7. The authors conclude that these interplanetary electrons are created simultaneously with the flare electrons which cause the microwave and X-ray burst. Probably only a small fraction of flare electrons escape into interplanetary space. In 1968 and 1969, Datlowe et al. (1969) with equipment on board OGO-5, and Koechlin et al. (1969) on the ESRO satellite HEOS A 1 measured on several occasions an excess flux of $>10 \mathrm{MeV}$ electrons in association with solar flares.

Evidence has been given that some fairly strong particle events recorded close to the Earth were due to flares situated on the invisible solar hemisphere. This seems to be the case with particles recorded on 1966 July 16-19, which Dodson et al. (1969) ascribe to a flare that occurred near the central meridian on the opposite solar hemisphere, in the active region which produced the July 7 cosmic ray flare; the most extraordinary event of this type, however, occurred on 28 January 1967 (Baird et al., 1967). On that day, a GLE was recorded by neutron monitors, without any obvious source on the visible solar hemisphere. This fact, as well as combined measurements with space probes in different distances from the Earth-Sun line, and the unusual isotropy of the particle flux, give strong support to the origin of the particles far behind the solar limb (Innanen et al., 1968; Bukata et al., 1969); a position $60^{\circ}$ behind the western limb seems to be the most probable one (Dodson and Hedeman, 1969b; Lockwood, 1968). An interesting feature of the event also was a low-energy precursor of the cosmic-ray enhancement (Blake et al., 1969; Masley, 1969).

Many papers have been published on proton measurements during individual particle events in space, and the most detailed studies were presented by Lindgren (1968) and Lanzerotti (1969) on the 1967 May 24 and 28, events. Several selected events and periods were studied in an international cooperation, which has greatly improved the possibility to get a fairly complex picture of the solar event and its consequences in space. These were the events of 1966 July 7, (results published in Annals IQSY, Vol. 3), 1966 August 28 and September 2 (Švestka and Simon, 1969), 1967 May 23 (Lincoln, 1969), and 1969 February 25 (Third ESRIN/ESLAB Symposium at Noordwijk). The strongest event during this period was that of 1966 September 2. Dosage of $\sim 60 \mathrm{R} / \mathrm{h}$ behind a $0.22 \mathrm{~g} / \mathrm{cm}^{2}$ thick aluminium wall was measured during its maximum phase (Kane and Winckler, 1969), and it also was the fifth case, the first one after 1961, when nuclei with charges greater than 2 were detected in the particle flux (Durgaprasad et al., 1968).

The acceleration of charged particles to high energies on the Sun has stimulated the search for high energy neutrons also associated with flares. OSO 1 satellite results (Hess and Kaifer, 1967) have failed to show any neutron effects associated with specific flare activity. A positive flux at the Earth of $\sim 10^{-1}$ neutrons $/ \mathrm{cm}^{2} \mathrm{~s}$ for $50-500 \mathrm{MeV}$ range has been reported by Daniel et al. (1967) in association with a subflare, but Holt (1967), on the base of negative OGO 1 measurements, has raised serious doubt as to whether such a high flux was possible without the detection of decay protons in interplanetary space. No increase higher than $10^{-2}$ neutrons $/ \mathrm{cm}^{2} \mathrm{~s}$ has been found by Zych and Frye (1969) for periods after two flares of importance 1, and by Daniel et al. (1969) 
after a 2B-class flare. Of course, no strong particle events have been investigated in this way, and theoretical considerations indicate that a measurable neutron flux could still be detected after such events as that of 1966 September 2 (Lingenfelter and Ramaty, 1967; Lingenfelter, 1969).

\section{SOLAR WIND DISTURBANCES}

(by J. M. Wilcox, Berkeley)

Review papers on solar wind plasma and magnetic fields were written by Dessler (1968), Wilcox, (1968), Axford (1968), Hundhausen (1968), and Parker (1969). Solar wind plasma observations included electrons and several ions not previously observed. Solar wind electrons (Montgomery et al., 1968; Formisane, 1969) were observed to have temperatures always greater than or equal to proton temperature, with the range between $7 \times 10^{4}$ and $2 \times 10^{5} \mathrm{~K}$. A two fluid model of the solar wind (Hartle and Sturrock, 1968) showed that the collision energy exchange rate between electrons and protons is sufficiently slow that electrons and protons can have quite different temperatures. The average helium abundance in the solar wind (Ogilvie and Wilkerson, 1969) was observed to be approximately $0 \cdot 05$, suggesting that the solar abundance may be about half the previously quoted estimates, while helium abundances in the range of 0.15 observed for a few hours at a time may have been associated with the driver gas of a solar flare-induced shock wave. Hundhausen et al. (1968) compared the observed flux of various solar wind oxygen species and determined the temperature of the coronal region from which the solar wind came, since the ionization state of the expanding coronal plasma is established deep in the corona and remains unchanged in transit through interplanetary space.

Hundhausen et al. (1967) observed the quiet solar wind velocity to be near $320 \mathrm{~km} / \mathrm{s}$ with variations between 280 and $750 \mathrm{~km} / \mathrm{s}$. Proton temperatures ranged from $8 \times 10^{5}$ to $6 \times 10^{3} \mathrm{~K}$. An east-west asymmetry in the solar wind velocity caused by fast stream-slow stream interaction was observed by Siscoe et al. (1969), and a sector-dependent asymmetry in the north-south component of the solar wind flow was inferred by Siscoe and Coleman (1969). Siscoe and Finley (1969) used steady-state hydrodynamic equations to determine what pressure perturbations near the Sun are required to produce at Earth the observed north-south deviations from radial flow. Heating of collisionless solar wind plasma by damping of hydromagnetic waves was discussed by Barnes (1969), and heating by long wavelength turbulence was discussed by Jokipii and Davis (1969).

The large-scale sector structure continued to be a prominent feature of the interplanetary magnetic field (Wilcox and Colburn, 1969). The formation of a new interplanetary sector was related to development of a solar active region that produced magnetic loops in the interplanetary medium by Schatten et al. (1968). Wilcox and Howard (1968) compared interplanetary and photospheric magnetic fields and postulated a solar sector pattern with sector boundaries at almost constant longitude in a latitude range from $40^{\circ} \mathrm{N}$ to $40^{\circ} \mathrm{S}$. A correlation between right-handed polarization of the solar continuum radial emission and away polarity interplanetary magnetic sectors was observed by Krüger et al. (1968). A model for the transition between coronal and interplanetary magnetic fields was proposed by Schatten et al. (1969), and the solar origin of the interplanetary magnetic field was also studied by Wilcox and Ness (1967), Winters et al. (1969), and Wilcox et al. (1969). Carovillano and Siscoe (1969) with approximate solutions to the hydromagnetic equations were able to find reasonable agreement with sector structure observations. Bumba and Obridko (1969) have shown that flares are most probable close to solar sector boundaries. Svestka (1968d) and Lanzerotti (1969) have found that in association with some flare events energetic particles are stored within the range of a sector boundary, and with decreasing energy tend to accumulate toward and behind the eastern boundary limit.

Solar flares produce interplanetary shock waves that are decelerated as they expand and propagate through the quiescent solar wind plasma (Dryer and Jones, 1968; Hirshberg, 1968; Ogilvie et al., 1968; Hundhausen and Gentry, 1969; Ness and Taylor, 1969; Ogilvie and Burlaga, 1969). All clearly defined ssc's at the Earth are caused by hydromagnetic shocks (Burlaga and Ogilvie, 1969), but generally a ssc or si is often the result of tangential discontinuities in the solar wind, and does 
not imply the existence of a shock wave (Gosling et al., 1967). Levy et al. (1969) observed Faraday rotation of the telemetry signal from Pioneer 6 when it was occulted by the Sun. Transient magnetic effects were related to limb flares (Schatten, 1970) and the large-scale Faraday rotation was consistent with the interplanetary magnetic sector structure.

\section{SOLAR-TERRESTRIAL RELATIONS}

(extracted from a draft* prepared by C. Sawyer, Boulder)

The relative dearth of very energetic particle events (GLE) at the maxima of cycles 18 and 19 has not been observed in cycle 20 (Dodson and Hedeman, 1969b). This identifies the inhibition of these events with a critical level of activity, rather than with a particular phase of the cycle (Švestka, 1969). Another difference in the new cycle, perhaps due to the relatively low solar activity and unusually stable interplanetary conditions, is the identification of particle emission with flares on the invisible hemisphere of the Sun (cf. Section 15).

Several authors have continued studies on small-scale statistical increases in cosmic ray intensity after flares of smaller importance. Increases of about $0.1 \%$ at balloon altitudes have been found, larger for flares located west of central meridian and at low heliographic latitude (Adagarova et al., 1968; Vladimirskij, 1968). Evidence for weaker solar and interplanetary magnetic fields at high heliographic latitudes comes from the semi-diurnal wave in cosmic-ray intensity (Lietti and Quenby, 1968; Patel et al., 1968). An index of cosmic ray intensity and so of solar activity over periods $>10^{5}$ yr may be provided by measurement of ${ }^{59} \mathrm{Ni}$ in dated sedimentary layers (Hasegawa et al., 1968).

Geomagnetic disturbance most often occurs 2 or 3 days after a sector boundary sweeps past the earth, while the arrival at the Earth of the boundary follows 4 or 5 days after central meridian passage of the corresponding photospheric boundary (Wilcox, 1968). Weak and transient disturbances, however, sometimes can be directly associated with the boundary crossing at the Earth (Švestka, 1968d). Roosen (1969) has shown that recurrent high values of the geomagnetic character figure C9 follow 4 days after CMP of solar regions with low radio emission in the microwave region. These "coronal suppressions" are supposed to be associated in a solar-wind model with enhanced temperature and velocity, diminished density, and low polytropic index; the low density leads to the observed low radio brightness temperature.

The time sequence and interrelations among variations of the indexes $D_{s t}, A_{E}$, and PCA are consistent with the hypothesis that the solar "storm cloud" may contain a large flux of protons with energy in the 10 to $100 \mathrm{keV}$ range, and that geomagnetic effects are explained by these solar particles, with no necessity for acceleration or storage in the magnetosphere (Haurwitz, 1969a). This hypothesis is consistent with enhancement of polar-glow (optical) aurora at the time of ssc (Sanford, 1967), and with subsequent direct observations of large and variable flux of particles in the energy range outside and within the magnetosphere (Frank, 1967). Arguments are advanced for interpreting the observed north-south asymmetry of solar activity and its terrestrial consequences entirely in terms of solar radiation, with no asymmetry in the propagation of the particles that cause ssc, PCA or GLE (Popovici and Maris, 1968; Maris and Dinulescu, 1969). Ballario (1967) has studied the history of active regions in relation to geomagnetic disturbances and distinguishes different types of plages, which are associated with disturbed and quiet days, respectively. Haurwitz (1969b) has shown that the period of Pi2 micropulsations may offer a means of devising terrestrial indices that would separate the magnitude of the solar stimulus from the sensitivity of the Earth's response. Extensive lists of well-defined geomagnetic storms of sporadic and of recurrent type have been compiled by Mustel (1968).

From studies of ionospheric effects of flares, especially SFD which shows F-region effects (Donnelly, 1967), or from incoherent-scatter measurements of electron density as a function of height and time (Garriott, 1967; Garriott et al., 1969), the relative enhancement within several broad wave-

* A vailable on request. 
length ranges can be estimated. Line and continuum radiation in the 500 to $1000 \AA$ range is found to be the major contributer to the SFD. Ohshio is using the fluctuation of signal strength at $25 \mathrm{kHz}$ to derive a daily and monthly index presumed to measure $0-3 \AA$ radiation. At the Max-Planck Institute for Aeronomy at Lindau, the solar Lyman $L \alpha$ and X-radiation are compared with D-layer absorption and E-layer critical frequency; $10-\mathrm{cm}$ flux, sunspot number and UV flux are compared with F-layer electron-density distribution and with temperature and density in the thermosphere. The Ionospheric Group at Leicester University collaborates with the X-ray group in comparing recorded SID effects with X-ray bursts. Organized efforts to observe ionospheric flare effects are sponsored by IUCSTP, under the leadership of J. W. King.

A very thorough treatment of the influence of solar activity on the weather has been given by Schuurmans (1969). One can agree with W. O. Roberts' opinion that work in this field is going to become very important in the decade ahead. It is a field that has long suffered from a good deal of sloppy and uncritical work, and it is therefore good to have high-quality papers, as this one by Schuurmans, on the subject. This problem is also well treated in three review papers on solarterrestrial relations published by Mustel (1968), which are concerned with the quiet and disturbed solar wind and the influence of solar particle streams on the lower layers of the Earth's atmosphere. Mustel's studies of dependence of barometric pressure on solar activity, as described by geomagnetic disturbances, suggest that solar particles may contribute to heating the stratosphere and influence the circulation of the lower atmosphere.

Proceedings of a Symposium on Solar-Terrestrial Physics organized by the Sovet Solnce-Zemlja of the Soviet Academy of Sciences at Crimea in October 1968 have been edited by Obridko (1969). Gnevyshev is preparing a compilation of Soviet studies of solar activity effects in the biological sphere.

\section{BIBLIOGR APHY}

Acton, L. W. 1968, Astrophys. J., 152, 305.

Adagarova, Kh., Ivanov, V., Kolomeets, E. 1968, Can. J. Phys., 46, 5745.

Alexander, J. K., Malitson, H. H., Stone, R. G. $\quad$ 1969, Solar Phys., 8, 388.

Alexandrovich, I. I., Stepanov, V. E. 1969 , Izv. SibIZMIR, no. 2, in press.

Alfvén, H., Carlquist, P. $\quad$ 1967, Solar Phys., 1, 220.

Allen, C. W. $\quad 1969$, Solar Phys. 8, 72.

Altschuler, M. D. $\quad$ 1967, Solar Phys., 1, 377.

Altschuler, M. D., Nakagawa, Y., Lillienquist, C. G. $\quad$ 1968, Solar Phys., 3, 466.

Anderson, K. A. $\quad$ 1969, Solar Phys., 6, 111.

Anichini, M., Godoli, G. $\quad$ 1967, Mem. Soc. astr. ital., 38, 259.

Anzer, U. 1968, Solar Phys., 2, 298.

Anzer, U. $\quad$ 1969, Solar Phys., 8, 37.

Anzer, U., Tandberg-Hanssen, E. 1970, Solar Phys., 11, 61.

Arnoldy, R. L., Kane, S. R., Winckler, J. R. 1967, Solar Phys., 2, 171.

Arnoldy, R. L., Kane, S. R., Winckler, J.R. $\quad$ 1968a, IAU Symposium no. 35, p. 490; Astrophys. J., 151, 711.

Arnoldy, R. L., Kane, S. R., Winckler, J. R. 1968b, School of Physics and Astronomy, University of

Minnesota, CR-108.

Axford, W. I. 1968, Space Sci. Rev., 8, 331.

Babin, A. N. 1969, Izv. Krym. astrofiz. Obs., 41-42, in press.

Bachmann, G. $\quad 1969$, Astr. Nachr., 291, 131.

Baird, G. A., Bell, G. G., Duggal, S. P., Pomerantz, M. A. $\quad$ 1967, Solar Phys., 2, 491.

Ballario, M. C. $\quad 1967$, IQSY Notes 21, 36; 1969, Mem. Soc. astr. ital., in press.

Banin, V., de Feiter, L. D., Fokker, A. D., Martres, M. J., Pick, M. 1969, Ann. IQSY, 3, 22.

Baranovskij, E. A. 1969, Izv. Krym. astrofiz. Obs., 40, 41, in press.

Barnes, A. $\quad 1969$, Astrophys. J., 154, 751; 155, 311.

Becker, R. A. $\quad 1969$, Space Physics at the Aerospace Corporation, El Segundo, California.

Beckers, J. M. $\quad 1968$, Solar Phys., 3, 258.

Beckers, J. M. 1968 b Solar Phys., 5, 15.

Beckers, J. M. 1969, Solar Phys., 9, 372. 
Beckers, J. M., Schröter, E. H. Beckers, J. M., Schröter, E. H. Beckers, J. M., Schröter, E. H. Beckers, J. M., Schröter, E. H. Beckers, J. M., Stenflo, J. O. Beckers, J. M., Tallant, P. E. 1968a, Solar Phys., 4, 142.

1968b, Solar Phys., 4, 303.

1969a, Solar Phys., 7, 22.

1969b, Solar Phys., in press.

1969, Solar Phys., 6, 480.

1969, Solar Phys., 7, 351.

Beigman, I. L., Grineva, Yu. I., Mandelshtam, S. L., Vainstein, L. A., Zhitnik, I. A. I969, Solar Phys., 9, 160.

Bigg, E. K., Mulhali, P. S. $\quad$ 1967, Proc. astr. Soc. Australia, 1, 53.

Blake, J. B., Paulikas, G. A., Freden, S. C. 1969, Symposium on Solar Flares and Space Research, p. 258.

Blizard, J. B. 1969, Paper presented at the 15th Annual AAS Meeting, Denver.

Bohlin, J. D., Simon, M. 1969, Solar Phys., 9, 183.

Böhme, A., Krüger, A. 1968, HHI Suppl. Ser. Solar Data, 1, 51.

Boischot, A., Clavelier, B. $\quad 1968 a$, IAU Symposium no. 35, p. 565.

Boischot, A., Clavelier, B. $\quad$ 1968b, Ann. Astrophys., 31, 445.

Boischot, A., Daigne, G. 1968, Ann. Astrophys., 31, 531.

Branch, D. 1970 , Astrophys. J. 159, in press.

Bray, R. J. 1968, Solar Phys. 4, 318.

Broadfoot, A. L. 1967 , Astrophys. J., 149, 675.

Bruickner, G. $\quad 1968$, NRL Space Res. Seminar, NRL Washington, D.C., p. 27.

Bruzek, A. 1967, Solar Phys., 2, 451.

Bruzek, A. 1968, Nobel Symposium no. 9, p. 67.

Bruzek, A. $\quad 1969 a$, Symposium on Solar Flares and Space Research, p. 61.

Bruzek, A. $1969 b$, Solar Phys., 8, 29.

Bukata, R. P., Gronstal, P. T., Palmeira, R. A. R., McCracken, K. G., Rao, U. R. 1969, Solar Phys., $10,198$.

Bumba, V. $\quad 1967 a$, Plasma Astrophysics, Intern. School of Physics E. Fermi, 39, p. 77.

Bumba, V. $1967 b$, Solar Phys., 1, 371.

Bumba, V., Howard, R. F. 1969, Solar Phys., 7, 28.

Bumba, V., Obridko, V. N. 1969, Solar Phys., 6, 104.

Bumba, V., Howard, R. F., Martres, M. J., Soru-Iscovici, I. $\quad$ 1968, IAU Symposium no. 35, p. 13.

Bumba, V., Howard, R. F., Kopecký, M., Kuklin, G. $\quad$ 1969, Bull. astr. Inst. Csl., $20,18$.

Burlaga, L. F., Ogilvie, K. W. 1969, J. geophys. Res., 74, 2815.

Carlqvist, P. $\quad$ 1968, Nobel Symposium no. 9, p. 193, and 1969, Solar Phys., 7, 377.

Carovillano, R. V., Siscoe, G. L. $\quad$ 1969, Solar Phys., 8, 401.

Castelli, J. P., Aarons, J., Michael, G. A. 1967, J. geophys. Res., 72, 5491.

Chitre, S. M. 1968, Solar Phys., 4, 168.

Chitre, S. M., Shaviv, G. 1967, Solar Phys., 2, 150.

Cimino, M., Cacciani, A., Sopranzi, N. 1968, Solar Phys., 3, 618.

Clark, C. D., Park, W. M. 1968, Nature, 219, 922.

Cline, T. L., McDonald, F. B. 1968, Solar Phys., 5, 507; 1969, Ann. IQSY, 3, 205.

Cline, T. L., Holt, S. S., Hones, E. W. 1968, J. geophys. Res., 73, 433.

Covington, A. E., Legg, T. H., Bell, M. B. 1967, Solar Phys., 1, 465.

Croom, D. L., Powell, R. J. 1969, Nature, 221, 945.

D'Angelo, N. 1969, Solar Phys., 7, 321.

Daniel, R. R., Joseph, G., Lavakare, P. J., Sunderrajan, R. 1967, Nature, 213, 21.

Daniel, R. R., Gokhale, G. S., Joseph, G., Lavakare, P. J., Sekhon, B. S. $\quad$ 1969, Solar Phys., $10,465$.

Datlowe, D. W., L'Heroux, J., Meyer, P. 1969, Paper presented at the 11th Int. Conf. on Cosmic Rays. Budapest.

De Groot, T., Van Nieuwkoop, J. $\quad$ 1968, Solar Phys., 4, 332.

De Jager, C. $\quad$ 1968, Nobel Symposium no. 9, p. 171.

De Jager, C., Svestka, Z. (eds.) 1969, Solar Flares and Space Research, 11th Plenary Meeting of COSPAR Symposium, North-Holland Publ. Comp., Amsterdam.

Demarq, J., Rösch, J. 1967, Adv. opt. Techn., 7.

De Mastus, H. L., Stover, R. R. 1967, Publ. astr. Soc. Pacific, 79, 615.

Dessler, A. J. $\quad$ 1967, Rev. Geophys., 5, 1.

Deubner, F. L. $\quad$ 1968, IAU Symposium no. 35, p. 230. 
Deubner, F. L. $\quad$ 1969, Solar Phys., 7, 87.

Deubner, F. L., Liedler, R. 1969, Solar Phys., 7, 87.

Dizer, M. 1968, Solar Phys., 4, 99.

Dizer, M. 1969, Solar Phys., 10, 416.

Dodson, H. W. 1969, Ann. IQSY, 3, 21.

Dodson, H. W., Hedeman, E. R. $\quad 1968 a, I A U$ Symposium no. 35, p. 56.

Dodson, H. W., Hedeman, E. R. $\quad 1968 b$, Nobel Symposium no. 9, p. 37.

Dodson, H. W., Hedeman, E. R. $\quad$ 1968c, Solar Phys., 4, 229.

Dodson, H. W., Hedeman, E. R. 1969a, World Data Center A - Report UAG - 5, 7.

Dodson, H. W., Hedeman, E. R. $\quad$ 1969b, Solar Phys., 9, 278.

Dodson, H. W., Hedeman, E. R., Kahler, S. W., Lin, R. P. $\quad$ 1969, Solar Phys., 6, 294.

Donnelly, R. 1967, J. geophys. Res., 72, 5247; 1969, ibid. 74, 1873.

Drake, J. F., Gibson, J., Van Allen, J. A. $\quad$ 1969, Solar Phys., 10, 433.

Dryer, M., Jones, D. L. $\quad$ 1968, J. geophys. Res., 73, 4875.

Durgaprasad, N., Fichtel, C. E., Guss, D. E., Reames, D. V. $\quad$ 1968, Astrophys. J., $154,307$.

Eddy, J. A., Malville, J. M. 1968, Astrophys. J. 150, 289.

Eliseeva, L. A. $\quad$ 1967, Izv. Krym. astrofiz. Obs,, 37, 153.

Elliot, H. 1969, Symposium on Solar Flares and Space Research, p. 356.

Elwert, G. 1968, Mitt. astron. Ges., 25, 201.

Engvold, O. 1969, Solar Phys., 8, 284.

Engvold, O., Maltby, P. $\quad$ 1968, Nobel Symposium no. 8, p. 109.

Énomé, S., Kakinuma, T., Tanaka, H. 1969, Solar Phys. 6, 428.

Eriksen, G., Maltby, P. 1967, Solar Phys., 2, 249.

Evans, K., Pounds, K. A. 1968, Astrophys. J., 152, 319.

Evans, K., Balstrone, R., Parkinson, J., Pounds, K. A. $\quad 1969$, Solar Phys., in press.

Falciani, R., Rigutti, M. 1967, Observatory, 87, 170.

Falciani, R., Righini, A., Rigutti, M. 1967, Z. Astrophys., 67, 481.

Fan, C. Y., Pick, M., Pyle, R., Simpson, J. A., Smith, D. R. $\quad$ 1968, J. geophys. Res., 73, 1555.

Fokker, A. D. 1967, Solar Phys., 2, 316.

Formisano, V. 1969, J. geophys. Res., 74, 355.

Fortini, T., Torelli, M. 1968, IAU Symposium no. 35, p. 50.

Frank, L. A. 1967, J. geophys. Res., 72, 3753.

Frazier, E. N., Sherrer, P. H. 1969, Solar Phys., 10, 297.

Fredga, K. 1969, Solar Phys., 9, 358.

Friedman, H. 1969, Symposium on Solar Flares and Space Research, p. 87.

Friedman, H., Kreplin, R. W. $\quad$ 1969, Ann. IQSY 3, 78, 144.

Friedman, M., Hamberger, S. M. 1968, Astrophys. J., 152, 667.

Friedman, M., Hamberger, S. M. 1969, Solar Phys., 8, 104.

Fritzová-Švestková, L., Švestka, Z. 1967, Solar Phys., 2, 87.

Frost, K. 1969, Astrophys. J. Letters, 158, in press.

Gabriel, A. H., Jordan, C. 1969, Nature, 221, 947, and Mon. Not. R. astr. Soc., in press.

Gaizauskas, V. 1969, Report presented to the Pasadena Meeting of the A.A.S. in February 1969.

Garriott, O. 1967, J. geophys. Res., 72, 6099.

Garriott, O., da Rosa, A., Davis, M., Wagner, L., Thome, G. $\quad$ 1969, Solar Phys., 8, 226.

Getling, A. V., Tverskij, B. A. 1967, Astr. Zu., 45, 606.

Gintzburg, M. A. 1967, J. geophys. Res., 72, 2749; 1968, Astr. Zu., 45, 610.

Gleissberg, W. 1967, Astr. Nachr., 290, 11.

Gnevyshev, M. N., Chistjakov, V. F. 1968, Soviet Astr. 12, no. 5.

Gnevyshev, M. N., Nikolskij, G. M., Sasanov, A. A. 1967, Solar Phys., 2, 223.

Godoli, G. 1967, Oss. Mem. Oss. astrofis. Arcetri, 87.

Godoli, G., Mazzucconi, F., Monsignori Fossi, B. C. $\quad$ 1967, Ann. Geophys., 19, 95; $20,183$.

Göhring, R. 1969, Solar Phys., 8, 271.

Gold, T. 1968, Nobel Symposium no. 9, 205.

Goldberg, L. $\quad$ 1967, A. Rev. Astr. Astrophys., 5, 279.

Gopasjuk, S. I. $\quad$ 1967, Izv. Krym. astrofiz. Obs., 38, 65; 1969, ibid., 40, 111.

Gopasjuk, S. I., Tsap, T. T. $1969 a$, Astr. J., 46; 1970, Izv. Krym. astrofiz. Obs., 43, in press.

Gopasjuk, S. I., Tsap, T. T. 1969b, Izv. Krym. astrofiz. Obs., 41-42, in press.

Gopasjuk, S.I., Erjushev, N.iN., Neshpor, Ju. I. $\quad$ 1967,Izv.Krym.astrofiz.Obs., 37, 109; 1969, ibid. $39,298$. 
Gosling, J. T., Asbridge, J. R., Bame, S. J., Hundhausen, A. J., Strong, I. B. $\quad$ 1967, J. geophys. Res., 72, 3357; 1968, ibid. 73, 43.

Grigorev, N. L., Lutsenko, V. N., Pisarenko, N. F., Savenko, I. A. 1969, Paper presented at the 11th Conf. on Cosmic Rays, Budapest.

Hall, L. A., Hinteregger, H. E. 1969, Symposium on Solar Flares and Space Research, p. 81.

Hansen, R., Hansen, S., Loomis, H. 1969 , Solar Phys., 10, 135.

Hartle, R. E., Sturrock, P. A. $\quad$ 1968, Astrophys. J., 151, 1155.

Hartz, T. R. 1969, Planet. Space Sci., 17, 267.

Harvey, J. W. 1969, Thesis, University of Colorado, Boulder.

Harvey, J., Livingston, W. 1969, Solar Phys., 10, 283.

Harvey, J. W., Tandberg-Hanssen, E. 1968, Solar Phys., 3, 316.

Hasegawa, H., Yamakoshi, K., Noma, M., Maihara, T. 1968, Canad. J. Phys., 46, 5930.

Haugen, E. $\quad 1969$, Solar Phys., 9, 88.

Haurwitz, M. W. 1968, Astrophys. J., 151, 351.

Haurwitz, M. W. 1969a, J. geophys. Res., 74, 2348.

Haurwitz, M. W. 1969 , Planet. Space Sci., in press.

Hearn, A. G., Noyes, R. W., Withbroe, G. L. $\quad$ 1969, Mon. Not. R. astr. Soc., 144, 351.

Henoux, J. C. $1968 a$, Solar Phys., 4, 315.

Henoux, J. C. 1968b, Ann. Astrophys., 31. 511.

Hirshberg, J. 1968, Planet. Space Sci., 16, 309.

Holt, S. S. 1967, J. geophys. Res., 72, 3507.

Holt, S. S., Cline, T. L. 1968 , Astrophys. J., 154, 1027.

Holt, S. S., Ramaty, R. 1969, Solar Phys., 8, 119.

Howard, R., Bumba, V., Smith, S. 1967, Atlas of Solar Magnetic Fields, Carnegie Inst. Washington, D.C. Publ. 626.

Howard, R., Tanenbaum, A. S., Wilcox, J. 1968, Solar Phys., 4, 286.

Hudson, H. S., Peterson, L. E., Schwartz, D. A. 1969a, Solar Phys., 6, 205; Astrophys. J., 157, 389.

Hudson, H., McKenzie, D., Zirin, H., Ingham, W. 1969b, 18-21 February 1969 AAS Meeting, Pasadena.

Hundhausen, A. J. $\quad$ 1968, Space Sci. Rev., 8, 690.

Hundhausen, A. J., Gentry, R. A. 1969, J. geophys. Res., 74, 2908.

Hundhausen, A. J., Asbridge, J. R., Bame, S. J., Strong, I. B. 1967, J. geophys. Res., 72, 1979.

Hundhausen, A. J., Gilbert, H. E., Bame, S. J. 1968 , J. geophys. Res., 73, 5485.

Hyder, Ch. L. $\quad$ 1967, Solar Phys., 2, 49, 267.

Hyder, Ch. L., Mauter, H. S., Shutt, R. L. 1968, Astrophys. J., 154, 1039.

Ikhsanov, R. N. 1967, Astr. Zu., 44, 1048, 1211.

Ikhsanov, R. N., Platonov, Y. P. 1967 , Soln. Dann., 11, 78.

Innanen, W. G., Krimigis, S. M., Armstrong, T. P. 1968, paper presented at the Midwest Cosmic Ray Conference, Iowa City.

Ioshpa, B. A. 1968, Soln. Aktivn., 3, 44.

Janssens, T. J., White, K. P. 1970, Solar Phys., in press.

Jensen, E., Brahde, R., Ofstad, P. 1969, Solar Phys., 9, 397.

Joffe, S. B., Smirnova, T. A. 1967, Astr. Zu., 45, 1037.

Jokipii, J. R. 1969, Paper presented at the 11th Conf. on Cosmic Rays, Budapest.

Jokipii, J. R., Davis, L., Jr. 1969 , Astrophys. J., 156, 1101.

Jokipii, J. R., Parker, E. N. 1969, Astrophys. J., 155, 777.

Jones, B. B. Freeman, F. F., Wilson, R. 1968, Nature, 219, 252.

Kai, K. $\quad 1968 a$, Publ. astr. Soc. Japan, 20, 140.

Kai, K. 1968b, Publ. astr. Soc. Japan, 20, 154.

Kai, K. 1969, Proc. astr. Soc. Austr., 1, 186.

Kai, K. 1970, Solar Phys., in press.

Kane, S. R., Winckler, J. R. 1969a, Solar Phys., 6, 304.

Kane, S. R., Winckler, J. R. $1969 b$, School of Physics and Astronomy, University of Minnesota, CR-134.

Kassinskij, V. V. 1967, Soln. Dann., 2, 59.

Kassinskij, V. V. $\quad 1969$, Soln. Dann., 4, 76; 5, 82.

Katzenstein, J. 1968, J. opt. Soc. Am., 58, 1348.

Kiepenheuer, K. O. (ed.) 1968a, IAU Symposium no. 35, Structure and Development of Solar Active Regions, D. Reidel, Dordrecht, The Netherlands. 
Kiepenheuer, K. O. $\quad 1968 b$, IAU Symposium no. 35, p. 3.

Kiepenheuer, K. O. $\quad 1968$, Nobel Symposium no. 9, p. 123.

Kjeldseth Moe, O. 1968a, Solar Phys., 4, 267.

Kjeldseth Moe, O. $\quad$ 1968b, IAU Symposium no. 35, p. 202.

Kjeldseth Moe, O., Maltby, P. 1969, Solar Phys., 8, 275.

Kleczek, J. 1968, IAU Symposium no. 35, p. 280.

Kleczek, J., Kuperus, M. 1969 , Solar Phys., 6, 72.

Kneer, F., Mattig, W. $\quad$ 1968, Solar Phys., 5, 42.

Koechlin, Y., Raviart, A., Tregner, L., Blaud, C. J., Degli Antoni, G., Dilworth, C., Maccagni, D., Tanzi, E. G. 1969, Paper presented at the 11th Conf. on Cosmic Rays, Budapest.

Kolpakov, P. E. $\quad 1968$, Izv. Krym. astrofiz. Obs., 38, 59.

Kopecký, M. 1969, Bull. astr. Inst. Csl., 20, 296.

Kopecký, M., Kuklin, G. V. 1969a, Bull. astr. Inst. Csl., 20, 22.

Kopecký, M., Kuklin, G. V. 1969b, Solar Phys., 6, 241.

Kopecký, M., Obridko, V. 1968, Solar Phys., 5, 362.

Kopecký, V. 1968, Bull. astr. Inst. Csl., 19, 362.

Kopp, R. 1968, Doctoral Thesis, Harvard University, Cambridge, Mass.

Kopp, R., Kuperus, M. 1968, Solar Phys., 4, 212.

Korchak, A. A. $\quad$ 1967, Dokl. A. N. SSSR, 173, 291; Nature, 213, 1209.

Kotov, V. A. 1969 , Izv. Krym. astrofiz. Obs., 41-52, in press.

Koval, A. N. 1967, Izv. Krym. astrofiz. Obs., 37, 62.

Koval, A. N., Severnyj, A. B. 1970, Solar Phys., in press.

Krat, V. A. 1968, Nobel Symposium no. 9, p. 89.

Krat, V. A. 1969 , Izv. glav. astr. Obs. Pulkovo, 185, in press.

Krimigis, S. M. 1969, Ann. IQSY, 3, 457.

Křivský, L. $\quad 1968$, IAU Symposium no. 35, p. 465.

Křivský, L. 1969, Solar Phys., 9, 194.

Křivský, L., Nestorov, G. 1968, Bull. astr. Inst. Csl., 19, 197.

Krüger, A., Lauter, E. A. (eds.) 1969, Geod. geoph. Veröff. R.II. H. 10.

Krüger, A., Bumba, V., Howard, R., Kleczek, J. $\quad$ 1968, Bull. astr. Inst. Csl., 19, 180.

Künzel, H. $\quad$ 1969, Astr. Nachr., 291, 265.

Kuperus, M. 1969, Space Sci. Rev., 9, 713.

Kuperus, M., Tandberg-Hanssen, E. $\quad$ 1967, Solar Phys., 2, 39.

Kusminykh, V. D., Sitnik, G. F. $\quad$ 1967, Mitt. Staatl. astr. Sternberg Inst., 147, 3 and 7.

Labrun, N. R., Smerd, S. F. $\quad$ 1968, Proc. astron. Soc. Australia, 1, 140.

Lacombe, C., Mangency, A. 1969, Astr. Astrophys., 1, 325.

Landini, M., Monsignori Fossi, B. C., Poletto, G., Tagliaferi, G. L. $\quad$ 1968, Solar Phys., 5, 546.

Lanzerotti, L. J. 1969, J. geophys. Res., 74, 2851.

Lee, R. H., Harvey, J. W., Tandberg-Hanssen, E. $\quad 1969$, Appl. Opt., in press.

Leighton, R. B. $\quad 1969$, Astrophys. J., 156, 1.

Lemaire, P. $\quad$ 1969, Astrophys. Letters, 3, 43, also in IAU Symposium no. 36.

Léna, P. 1969, Solar Phys., 7, 217.

Leroy, J. L. 1967, Ann. Astrophys., 30, 249.

Leroy, J. L. $\quad$ 1969, Solar Phys., 7, 221.

Levickij, L. S. 1967, Izv. Krym. astrofiz. Obs., 37, 137.

Levy, G. S., Sato, T., Seidel, B. L., Stelzried, C. T., Ohlson, J. E., Rusch, W. V. T. $\quad$ 1969, Science, 166, 596.

Lietti, B., Quenby, J. 1968, Canad. J. Phys., 46, 5942.

Lin, R. P. $\quad$ 1969, Paper presented at the 11th Int. Conf. on Cosmic Rays, Budapest, and 1970, Solar Phys., in press.

Lin, R. P., Anderson, K. A. $\quad$ 1967, Solar Phys., 1, 446.

Lincoln, J. V. (ed.) 1969, World Data Center A Report UAG-5.

Lindgren, S. T. 1968, Solar Phys., 5, 382.

Lingenfelter, R. E. 1969 , Solar Phys., 8, 341.

Lingenfelter, R. E., Ramaty, R. 1967, Inst. Geophys. Planet. Phys., UCLA, Publ. 595, 34.

Liszka, L. 1968, Nobel Symposium no. 9, p. 117.

Livingston, W. C. $\quad 1968$, Astrophys. J., 153, 929.

Livshitz, M. A. 1968, Soln. Aktivn. 3, 78. 
Lockwood, J. A. 1968, J. geophys. Res., 73, 4247.

Loughhead, R. E. 1968, Solar Phys., 5, 489.

Loughhead, R. E., Bray, R. J., Tappere, E. J., Winter, J. G. $\quad$ 1968, Solar Phys., 4, 185.

Makita, M. 1968, Solar Phys., 3, 557.

Maltby, P. $\quad 1968 a$, Solar Phys., 4, 96.

Maltby, P. $\quad 1968 b$, Solar Phys., 5, 3.

Malville, J. M. 1968, Solar Phys., 4, 323; 5, 236.

Malville, J. M., Tandberg-Hanssen, E. $\quad$ 1969, Solar Phys., 6, 278.

Maris, G., Dinulescu, V. 1969, Stud. si Cerci. Astron., 14, 45.

Martres, M. J. 1968, IAU Symposium no. 35, p. 25.

Martres, M. J., Michard, R., Soru-Iscovici, I., Tsap, T. $\quad$ 1968, IAU Symposium no. 35, p. 318; Solar Phys., 5, 187.

Masley, A. J. $\quad$ 1969, Symposium on Solar Flares and Space Research, p. 279.

Mattig, W. 1969a, Solar Phys., 6, 413.

Mattig, W. 1969b, Solar Phys., 8, 291.

Mattig, W., Mehltretter, J. P. $\quad$ 1968, IAU Symposium no. 35, p. 187.

McCracken, K. G. $\quad$ 1969, Symposium on Solar Flares and Space Research, 202.

McIntosh, P. S. $\quad 1969$, Ann. IQSY, 3, 40.

McKenna, S. M. P. 1967, Astrophys. J., 150, 1087.

McKenna-Lawlor, S. M. P. $\quad 1968$, Astrophys. J., 153, 367.

McKenna-Lawlor, S. M. P. $\quad 1969$, Astrophys. J., in press.

McQueen, R. M. 1968, Appl. Opt., 7, 1149.

Meekins, J. F., Kreplin, R. W., Chubb, T. A., Friedman, H. $\quad$ 1968, Science, 162, 891.

Mehltretter, J. P. 1969, Solar Phys., 9, 387.

Memeth, T. 1967, Geofiz. Közl. Magyar, 16, No. 1-2.

Menzel, D. H. 1968, Nobel Symposium no. 9, p. 183; 1969, Symposium on Solar Flares and Space Research, p. 363.

Meyer, F. 1968, IAU Symposium no. 35, p. 485.

Michard, R. 1968, IAU Symposium no. 35, p. 307.

Monsignori Fossi, B. C., Poletto, G., Tagliaferri, G. L.

Research vol. 10, p. 196.

Monsignori Fossi, B. C., Poletto, G., Tagliaferri, G. L. $\quad$ 1969, Solar Phys., 10, 196.

Montgomery, M. D., Bame, S. J., Hundhausen, A. J. 1968, J. geophys. Res., 73, 4999.

Moreton, G. E., Severnyj, A. B. 1968, Solar Phys., 3, 282.

Mustel, E. R. $\quad$ 1968, Astr. Sov. Akad. Nauk. SSSR Sci. Inf., 10.

Nakagawa, Y., Swartztrauber, P. 1969, Astrophys. J., 155, 295.

Ness, N. F., Taylor, H. E. $\quad 1969$, Ann. IQSY, 3, 336.

Neupert, W. M. 1967, Solar Phys., 2, 294.

Neupert, W. M. 1969, A. Rev. Astr. Astrophys., 7, 121.

Neupert, W. M., Swartz, M. 1969, Phys. Rev. Letters, in press.

Neupert, W. M., Gates, W., Swartz, M., Young, R. 1967, Astrophys. J., 149, L79.

Neupert, W. M., White, W. A., Gates, W. J., Swartz, M., Young, R. M. $\quad$ 1969, Solar Phys., 6, 183.

Newkirk, G. 1967a, Planet. Space Sci., 15, 1267.

Newkirk, G. 1967b, A. Rev. Astr. Astrophys., 5, 213.

Newkirk, G., Hansen, R. T., Hansen, S. 1969, Ann. IQSY, 3, 49.

Nikonov, O. V., Nikonova, E. S. 1969 , Soln. Dann., 4, 97; 5, 86.

Nikulin, N. S. $\quad 1967$, Izv. Krym. astrofiz. Obs., 36, 76.

Noyes, R. W., Goldberg, L., Parkinson, W. H., Reeves, E. M., Withbroe, G. L. $\quad$ 1968, Astr. J., 73, 573.

Noyes, R. W., Withbroe, G. L., Kirshner, R. P. 1969, Conf. on the Chromosphere-Corona Transition Region, HAO Boulder.

Obridko, V. N. 1968a, Soln. Dann., 4, 68.

Obridko, V. N. 1968b, Bull. astr. Inst. Csl., 19, 183, 186.

Obridko, V. N. (ed.) 1969, Solnechno-zemnaja fizika 1, Akad. Nauk. SSSR, Sovet Solnce-Zemlja.

Ogilvie, K. W., Burlaga, L. F. $\quad$ 1969, Solar Phys., 8, 422.

Ogilvie, K. W., Burlaga, L. F., Wilkerson, T. D. 1968, J. geophys. Res., 73, 6809.

Ogilvie, K. W., Wilkerson, T. D. $\quad 1969$, Solar Phys., 8, 435.

Ogir, M. B. 1967, Izv. Krym. astrofiz. Obs., 36, 69; 37, 94.

Ogir, M. B. $\quad 1969$, Izv. Krym. astrofiz. Obs. 41-42, and 43, in press. 
Ohman, Y. (ed.) 1968, Mass Motions in Solar Flares and Related Phenomena, Nobel Symposium no. 9. Öhman, Y., Hosinsky, G., Kusoffsky, U. $\quad$ 1968, Nobel Symposium no. 9, p. 95.

Oster, L. $\quad$ 1968, Solar Phys., 3, 543.

Pande, M. C., Gaur, V. P., Tripathi, B. M. 1969, Solar Phys., 7, 370.

Parker, E. N. 1969, Space Sci. Rev., 9, 325.

Parks, G. K., Winckler, J. R. $\quad$ 1969, Astrophys. J., 155, L117.

Patel, D., Sarabhai, V., Subrahmanian, G. $\quad$ 1968, Can. J. Phys., 46, 5981.

Pintér, S. 1969, Solar Phys., 8, 149.

Pneuman, G. W. 1967, Solar Phys., 2, 462.

Pneuman, G. W., Kopp, R. $\quad 1970$, Solar Phys., in press.

Polonskij, W. W. 1967, Astr. Cirk., 416, 1.

Polupan, P. N. 1968, Soln. Aktivn., 3, 125.

Popovici, C., Maris, G. $\quad$ 1968, Stud. si Cerc. de Astron., 13, 3.

Popovici, C., Dimitriu, A. 1969, Ann. IQSY, 3, 31.

Porfirov, V. (ed.) Voprosy Astrofiziky, Izdatelstvo Naukove Dumka Kiev.

Porsche, H. 1968, Mitt. astron. Ges., 25, 55.

Rachkovskij, D. N. $\quad 1967 a$, Izv. Krym. astrofiz. Obs., 36, 9, 56.

Rachkovskij, D. N. $\quad 1967 b$, Izv. Krym. astrofiz. Obs., 36, 51.

Raju, P. K. $\quad 1968$, Mon. Not. $R$. astr. Soc., 139, 479.

Ramaty, R., Lingenfelter, R. E. 1968, Solar Phys., 5, 531.

Ramsey, H. E., Smith, S. F., Angle, K. L. 1968, Final Report High Resolution Solar Photography, LMSC-681495.

Rao, U. R., Allum, F. R., Bartley, W. C., Palmeira, R. A. R., Harries, J. A., McCracken, K. G. 1969, Symposium on Solar Flares and Space Research, p. 267.

Rayrole, J. 1967, Ann. Astrophys. 30, 257.

Rees, D. E. 1969, Solar Phys., 10, 268.

Reidy, W. P., Vaiana, G. S., Zehnpfennig, T., Giaconni, R. $\quad$ 1968, Astrophys. J., 151, 333.

Rompolt, B. $\quad 1967$, Acta Astr., 17, 329.

Roosen, J. 1969, Solar Phys., 7, 448.

Ruhm, H. 1969, Solar Phys., 10, 104.

Rust, D. M. $\quad$ 1967, Astrophys. J., 150, 313.

Saito, M., Kato, S. $\quad$ 1968, Solar Phys., 3, 531.

Sakurai, K. 1967a, Rep. Iono. Space Res. Japan, 21, 29.

Sakurai, K. $\quad$ 1967b, Rep. Iono. Space Res. Japan, 21, 113.

Sakurai, K. $\quad$ 1967c, Rep. Iono. Space Res. Japan, 21, 213.

Sanford, B. $\quad$ 1967, Space Res., vol. 7, p. 836.

Sattarov, I. 1968, Soln. Dann., 8, 76.

Schatten K. H. 1970, Solar Phys., in press.

Schatten K. H., Ness, N. F., Wilcox, J. M. 1968, Solar Phys., 5, 240.

Schatten K. H., Wilcox, J. M., Ness, N. F. 1969, Solar Phys., 6, 442.

Schatzman, E. 1967, Solar Phys., 1, 411.

Schmidt, H. U. $\quad 1968, I A U$ Symposium no. 35, p. 95.

Schmidt, H. U. 1969, Symposium on Solar Flares and Space Research, p. 331.

Schröter, E. H., Wiehr, E. $\quad$ 1968, Mitt. astr. Ges., 25, 187.

Schuurmans, C. J. E. 1969, K. Ned. Meteorol. Inst. Med. Verhandl. no. 92.

Severnyj, A. B. $\quad 1967$, Izv. Krym. astrofiz. Obs., 36, 22.

Severnyj, A. B. $\quad 1968$ a, Izv. Krym. astrofiz. Obs., 38, 3.

Severnyj, A. B. $\quad 1968 b$, Nobel Symposium no. 9, p. 71.

Severnyj, A. B. $\quad 1968 c$, IAU Symposium no. 35, p. 233.

Severnyj, A. B. $\quad 1969 a$, Symposium on Solar Flares and Space Research, p. 38.

Severnyj, A. B. $1969 b$, Ann. IQSY, 3, 11 .

Severnyj, A. B., Steshenko, N. V. 1969, STP Notes, in press.

Severnyj, A. B., Zvereva, A. M. $\quad$ 1969, Izv. Krym. astrofiz. Obs., 41-42, in press.

Sheeley, N. R. 1967, Solar Phys., 1, 171.

Sheeley, N. R. 1969, Solar Phys., 9, 347.

Shimabukuro, F. I. 1968, Solar Phys., 5, 498.

Simon, G. W., Weiss, N. O. $1968, I A U$ Symposium no. 35, p. 108.

Simon, P., Svestka, Z. $\quad$ 1969, Ann. IQSY, 3 , 469. 
Simon, P., Martres, M. J., Legrand, J. P. 1969, Symposium on Solar Flares and Space Research, p. 405.

Siscoe, G. L., Coleman, P. J., Jr. $\quad$ 1969, Solar Phys., 8, 415.

Siscoe, G. L., Finley, L. T. 1969 , Solar Phys., 9, 452.

Siscoe, G. L., Goldstein, B., Lazarus, A. J. $\quad$ 1969, J. geophys. Res., 74, 1759.

Sitnik, G. F., Mitropolskaja, O. N. 1968, Astr. Zu., 45, 456.

Slonim, J. M. $\quad$ 1968, Astr. Zu., 45, 286, 726.

Slonim, J. M. $\quad 1969$, Astr. Zu., 46, 570, 697.

Slysh, V. I. $\quad 1967$, Astr. Zu., 44, 487; Kosm. Issled., 5, 897.

Smerd, S. F. $\quad$ 1968, Proc. astr. Soc. Austr., 1, 124.

Smith, E. v. P. $\quad 1968$, Nobel Symposium no. 9, 137.

Smith, E. v. P. $\quad$ 1969, Bull. Am. astr. Soc., 1, 262.

Smith, S. F., Howard, R. 1968, IAU Symposium no. 35, p. 33.

Smith, S. F., Ramsey, E. $\quad 1967$, Solar Phys., 2, 158.

Smolkov, G. J. 1969, Issl. geomagn. aeron., fizika Solnca, in press.

Smolkov, G. J., Bashkirtzev, V. S. 1969 , Issl. geomagn. aeron., fizika Solnca, in press.

Staude, J. 1969, Solar Phys., 8, 264.

Steenbeck, M., Krause, F. $\quad$ 1969, Astr. Nachr., 291, 49.

Stellmacher, G. 1969, Astr. Astrophys., 1, 62.

Stenflo, J. O. 1968a, Medd. Lunds astr. Obs. Ser. I, no. 233 and Ser. II, nos. 152 and 153.

Stenflo, J. O. 1968b, Solar Phys., 3, 482.

Stenflo, J. O. 1969a, Solar Phys., 8, 115.

Stenflo, J. O. 1969b, Solar Phys., 8, 260.

Stenflo, J. O. $1969 c$, Solar Phys., 6, 476.

Stepanjan, N. N. $\quad 1969$, Astr. Zu., 46, 580.

Steshenko, N. V. $\quad$ 1968, Izv. Krym. astrofiz., Obs., 38, 52.

Steshenko, N. V. 1970, Izv. Krym. astrofiz. Obs., 43, in press.

Steshenko, N. V., Shcherbakov, A. G. 1969, Izv. Krym. astrofiz. Obs., 41-42, in press.

Sturrock, P. A. 1968, Proc. E. Fermi Int. Summer School of Physics, 39th Course: Plasma Astrophysics, p. 168, and IAU Symposium no. 35, p. 471.

Surkov, E. P. 1969, Soln. Dann., $5,91$.

Śvestka, Z. 1968a, Solar Phys., 4, 18.

Svestka, Z. 1968b, IAU Symposium no. 35, p. 513.

Švestka, Z. $\quad 1968$ c, Nobel Symposium no. 9, p. 17.

Švestka, Z. 1968d, Solar Phys., 4, 361.

Švestka, Z. $\quad 1969$, Space Research, in press; Proceedings of the 3rd ESLAB/ESRIN Symp., in press.

Švestka, Z., Simon, P. 1969, Solar Phys., 10, 3.

Sweet, P. $\quad$ 1969, A. Rev. Astr. Astrophys., 7, 149.

Syrovatskij, S. I. 1969, Symposium on Solar Flares and Space Research, p. 346.

Takahashi, K. 1968, Solar Phys., 3, 598.

Takakura, T. 1967, Solar Phys., 1, 304.

Takakura, T., Uchida, Y., Kai, K. 1968, Solar Phys., 4, 45.

Tallant, P. E. 1970 , Solar Phys., in press.

Tanaka, H., Kakinuma, T., Enomé, S. $1969 a$, Ann. IQSY, 3, 63.

Tanaka, H., Kakinuma, T., Énomé, S., Torii, C., Tsukiji, Y., Kobayashi, S. $\quad$ 1969b, Proc. Res. Inst. atm. Nagoya Univ., 16, 113.

Teplitskaja, R. B., Turchina, V. D. 1969, Astr. Zu., 46, 74.

Tousey, R. 1967, Astrophys. J., 149, 239.

Tsap, T. T. 1967, Izv. Krym. astrofiz. Obs., 39, 265.

Tsap, T. T. 1969, Izv. Krym. astrofiz. Obs., 41-42, in press.

Uchida, Y. $\quad$ 1968, Solar Phys., 4, 30.

Unsöld, A. 1968, Q.J.R. astr. Soc., 9, 294.

Vaiana, G. S., Reidy, W. P., Zehnpfennig, T., Van Speybroek, L. $\quad$ 1968, AScience, 161, 564.

Valníčk, B. 1967, Bull. astr. Inst. Csl., 18, 249.

Valniček, B. 1968, IAU Symposium no. 35, p. 282.

Valnizek, B., Godoli, G., Mazzucconi, F. 1969, Ann. IQSY, 3, 113.

Van Allen, J. A., Wende, C. D. $\quad$ 1969, J. geophys. Res., 74, 3046.

Vernov, S. N., Chudakov, A. E., Vakulov, P. V., Gorchakov, E. V., Kontor, N. N., Legachev, Yu, I., 
Ljubimov, G. P., Pereslegina, N. V., Timofeev, G. A. 1969, Paper presented at the 3rd ESRIN/ ESLAB Symp. at Noordwijk, Sept. 1969.

Vinokur, M. 1968, Ann. Astrophys., 31, 457.

Vitinskij, Ju. L. 1967, Izv. glavn. astr. Obs. Pulkovo, 24, no. 6.

Vitinskij, Ju. L. $\quad 1969 a$, Solar Phys., 7, 210.

Vitinskij, Ju. L. 1969 b, Izv. glavn. astr. Obs. Pulkovo, no. 186, in press.

Vitinskij, Ju. L., Kopecký, M. 1968, Izv. glavn. astr. Obs. Pulkovo, no. 184.

Vladimirskij, B. $\quad$ 1968, Izv. Krym. astrofiz. Obs., 38, 132.

Von Klüber, H. 1967, Mon. Not. R. astr. Soc., $137,297$.

Von Klüber, H. $\quad$ 1968, Observatory, 88, 45; Mon. Not. R. astr. Soc., 141, 469.

Vojhanskaja, N. F. $\quad$ 1967, Astr. Obs. Trudy, 24, 73.

Warwick, J. W. 1967, Astrophys. J., 150, 1081.

Weart, S. R., Zirin, H. $\quad$ 1969, Publ. astr. Soc. Pacific, 81, 270.

Webber, J. C. 1969, Thesis, Pasadena.

White, K. P., Janssens, J. T. $\quad 1970$, Solar Phys., in press.

Wiehr, E. $\quad 1968$, IAU Symposium no. 35, p. 259.

Wiehr, E. 1969, Solar Phys., 9, 225.

Wiehr, E., Stellmacher, G., Schröter, E. H. $\quad$ 1968, Astrophys. Letters, 1, 181 ; 1969, ibid. 3, 91.

Wilcox, J. M. 1968, Space Sci. Rev., 8, 258; Symposium on Solar Flares and Space Research, p. 294.

Wilcox, J. M., Colburn, D. S. $\quad$ 1969, J. geophys. Res., 74, 2388.

Wilcox, J. M., Howard, R. 1968, Solar Phys., 5, 64.

Wilcox, J. M., Ness, N. F. $\quad$ 1967, Solar Phys., 1, 437.

Wilcox, J. M., Severnyj, A. B., Colburn, D. S. 1969, Nature, 224, 353.

Wild, J. P. (ed.) 1967, The Culgoora radio heliograph, Proc. Instn. Radio El. Eng. Austr., 28, 277.

Wild, J. P. $\quad 1968$ a, Proc. astr. Soc. Austr., 1, 137.

Wild, J. P. $\quad 1968$, Australian Physicist, 5, 117.

Wild, J. P. $\quad 1969$ a, Proc. astr. Soc. Austr., 1, 181.

Wild, J. P. $\quad 1969 b$, Solar Phys., 9, 260.

Wild, J. P., Sheridan, K. V., Kai, K. 1968, Nature, 218, 536.

Wilson, P. R. 1968a, Solar Phys., 3, 243, 454.

Wilson, P. R. $\quad 1968 b$, Solar Phys., 5, 338.

Wilson, P. R. $\quad 1969 a$, Solar Phys., 9, 391.

Wilson, P. R. 1969b, Solar Phys., 10, 404.

Winters, J. V., Ballif, J. R., Jones, D. E. 1969, Solar Phys., 7, 278.

Wittmann, A. 1969, Solar Phys., 7, 366.

Wittmann, A., Schröter, E. H. $\quad$ 1969, Solar Phys., 10, 357.

Wöhl, H. 1969, Solar Phys., 9, 394.

Xanthakis, J. 1969, Solar Phys., 10, 168.

Yip, W. K. 1967, Austr. J. Phys., 20, 421.

Yun, H. S. 1968, Thesis, Indiana University.

Zhukov, L. V., Muzalevskij, Ju. S. $\quad$ 1969, Astr. Zu., 46, 600.

Zirin, H., Russo Lackner, D. 1969 , Solar Phys., 6, 86.

Zirin, H., Werner, S. 1967, Solar Phys., 1, 66.

Zirker, J. B. 1967, Solar Phys., 1, 204.

Zwaan, C. $\quad$ 1967, Solar Phys., 1, 478.

Zwaan, C. $\quad 1968$, A. Rev. Astr. Astrophys., 6, 135.

Zych, A. D., Frye, G. M. $\quad$ 1969, J. geophys. Res., 74, 3726. 